\title{
An Interview with Romualdo Abulad, SVD
}

\section{Emmanuel C. De Leon}

\begin{abstract}
In this interview, the readers get the chance to listen to one of the most significant Thomasian philosophers, and undoubtedly, the most prominent Kantian scholar of our country. His passion for teaching, writing, and truth is very evident in this informal chat. The interviewer highlights the intellectual biography of Romualdo Abulad, beginning from his childhood in Lucban, Quezon, his accidental shift to philosophy, his awakening from his Thomistic slumber, and his love affair with Immanuel Kant. A bibliography of leading Thomasian philosophers can also be found at the end of the interview.
\end{abstract}

Keywords: Abulad, Filipino philosophy, interview, Thomasian philosophers

\section{A Child of Lucban, Quezon}

Emmanuel De Leon (Tagapanayam): Magandang araw po, Br. Romy! Salamat po sa pagpapaunlak ng isang interbyu sa gitna ng marami ninyong ginagawa. Unang-una po, narito po ako ngayon upang tanungin kayo ukol sa mga alaala ninyo ng klase ng pamimilosopiya sa Unibersidad ng Santo Tomas at baka sakali po ay mayroon tayong maiulat na kongkretong pamana nito sa uri ng pamimilosopiya na mayroong tayo ngayon sa ating bansa.

Pero, bago po ang lahat, dito po sa inyong curriculum vitae na nadownload ko sa internet, sinasabing nagtapos po kayo ng elementarya sa Lucban Elementary School noong 1960 at ng sekondarya naman sa Lucban Academy noong 1964. Br. Romy, maaari po ba ninyong gunitain para sa amin ang inyong kabataan sa probinsiya ng Quezon? Ano pong nagugunita ninyo tungkol sa inyong paglaki sa bayan ng Lucban?

Romualdo Abulad: Ah, mukhang marami ano? Let me see. Saan ba ako magsisimula?

De Leon: Ilan po kayong magkakapatid?

(C) 2016 Emmanuel C. De Leon

http://www.kritike.org/journal/issue 18/de leon june2016.pdf

ISSN 1908-7330

$(c c)$ BY-NC-ND 


\section{INTERVIEW WITH ROMUALDO ABULAD}

Abulad: Okay! Tatlo kaming magkakapatid. Ako lamang ang lalaki, at ako'y nasa gitna. Actually, second husband ng nanay ko ang tatay ko. 'Yung first husband niya ay sundalo na namatay noong giyera. Kaya mayroon akong half-sister-'yung panganay sa amin. Pero, noong lumaki kami ay parang wala kaming pagkakaiba o distinction. Talagang ang pakiramdam namin ay $100 \%$ ang relationship namin bilang magkakapatid.

So, doon ako lumaki [sa Lucban, Quezon], doon ako nagElementarya, doon ako nag-High School. At, ang pinakamahalagang bahagi ng aking buhay ay noong madestino ang parents ko sa ibang lugar [laughs]. Kaya naiwan ako sa lola ko. Lola ko ang pumalit sa mother ko at saka kasama ko sa bahay 'yung pinsan ko. Very lax 'yung aking lola kaya noong High School talagang lubog ako sa barkada. Doon ko natikman ang kahalagahan [ng pakikipagbarkada]. Alam mo, ang pinakamasayang bahagi ng aking buhay ay 'yung High School-dahil sa barkada. Barkada ang number one.

De Leon: At kung tungkol naman po sa sistema ng edukasyon noon sa probinsiya, ano naman pong klase ang meron noon sa Lucban?

Abulad: Siguro, isang dahilan kaya okay naman ang aking Ingles grammatically ay dahil na rin doon. Pero, hindi ko na malaman kung ano at sino ang nagcontribute [sa kung ano ako ngayon]. Siguro nag-aaral din naman ako. Siguro okay din ang mga guro ko. May mga guro na hanggang ngayon ay naaalala ko pa. Magagaling silang guro. Pero, mayroon ding mga teachers na katulad ng mga teachers ko sa college na ang tendency ay magtextbook, magmemorya, at pagkatapos ay magtest; at pagkatapos ng test ay makakalimutan mo na lahat. Parang hindi mo alam kung may nangyari o walang nangyari. Kaya sa maraming taon kong pag-aaral, tatlong teachers ang maituturing kong nagpabago ng aking pananaw - swerte na ako dahil mayroon akong tatlo. Alam kong maraming estudyante ang hindi nakatagpo ng mga teachers na tulad ng tatlong ito.

De Leon: Sinu-sino po silang tatlo?

Abulad: I mean, si Dr. [Emerita] Quito sa pilosopiya, si Dr. [Josephine] Pasricha pagdating sa ... Pilosoper siya pero siya rin ang nagturo sa amin ng literatura. At magagaling ang aming mga teachers sa literature noong araw, kasama na si [Cristina] Jingjing Pantoja-Hidalgo. Ang gagaling ng mga teachers namin sa Ingles at literature noon. Pero, natatandaan kong si Pasricha ang nagturo sa akin ng literary criticism. And after that, parang alam ko na kung papaano iaapproach at kung paano ako magbabasa ng literature o ng essay. So, Pasricha was one of my most influential teachers. And then,

(C) 2016 Emmanuel C. De Leon

http://www.kritike.org/journal/issue 18/de leon june2016.pdf

ISSN 1908-7330

(cc) BY-NC-ND 
lately pagdating sa theology, itong ating Kardinal Chito Tagle ngayon. At least may tatlo akong [maituturing na impluwensiya]. And they are really first class.

De Leon: Noon pong kabataan ninyo sa elementarya at sekondarya, wala po kayong naging impluwensiya doon?

Abulad: Meron, meron. Pero, hindi syempre kasing [lakas ng impluwensiya ng tatlong nabanggit]. Syempre, General Education iyan eh. Sila siguro ang dahilan kung bakit hindi baluktot ang aking Ingles, tama naman ang aking Tagalog, at nagkaroon ako ng interest sa Mathematics -'yung teacher ko sa Geometry ay hindi ko makakalimutan. Pinabayaan niya ako sa Geometry kaya kahit iba't iba ang aking solusyon sa mga geometrical problems, nakita ko kung bakit ang Geometry ay napaka-creative na subject. At, 'yun ang nagbigay sa akin ng inspirasyon para magustuhan ang Mathematics. Kung hindi siguro ako nag-pilosoper eh nag-Mathematician ako [laughs].

\section{On the UST College of Science and the Use of Intuition}

De Leon: Mathematician? [laughs] May kaugnayan po 'yan sa tanong ko mamaya. Pero, paano naman po kayo napunta sa Unibersidad ng Santo Tomas? Nag-aral po kayo sa UST simula noong 1964 hanggang 1969. Maaari po ba ninyong ikwento kung paano ninyo napili ang UST bilang inyong papasukang paaralan?

Abulad: Eh kasi pagkatapos ng High School ay parang lahat gusto ko eh [laughs]. Kaya noong tanungin ako ng tatay ko kung anong gusto kong kunin [kurso sa kolehiyo], hindi ako makasagot agad. Kaya siya na mismo ang nagdesisyon para sa akin at sinabing "Magdodoktor ka!" [laughs] So, punterya ang [pag-aaral ng] Medicine, kaya sa UST. Kaya tinulungan niya akong mag-enrol sa UST. So, sa College of Science ako nagsimula, 'no?

De Leon: Ah, sa College of Science po pala kayo noong 1964?

Abulad: Two years ako sa College of Science. Kaya mayroon din akong interest sa science. Pero, after two years, ewan ko kung paano nangyari 'yun, pero natatandaan ko noong summer [ng taong iyon], sinabi ko sa parents ko na I will shift. Ayaw nila. Pero, alam mo ang ginawa kong reason? [laughs] Hindi lang dapat ang paalam ay "I will shift," dahil kung iyon lang hindi papayag ang parents ko. Sabi ko, "I will go to a seminary." [laughs] Eh "pag seminaryo hindi makahindi ang mga magulang [laughs]. Diyos eh, Diyos [laughs]. 


\section{INTERVIEW WITH ROMUALDO ABULAD}

De Leon: Tama po. Lalo na po sa probinsiya ng Quezon.

Abulad: Right! Lalo sa aming bayan. It is very famous place para sa mga priests.

De Leon: Opo. Halos lahat ng mga pamilya kailangang may kahit isang pari.

Abulad: Totoo ka diyan, totoo ka diyan. So, walang magawa [ang parents ko]. Ang nanay ko naman ... teachers kasi silang pareho. Sila ang main influence ko. Parang nasa dugo ang pagiging teacher sa amin. So, by nature yata, teacher ako [laughs]. You can take away from me everything, pero teacher pa rin ako. By nature, teacher ako; tapos ang influence pa ng parents ko nga. They were very good teachers.

De Leon: Pagkatapos naman po ng dalawang taon sa College of Science dito sa UST, nagshift naman po kayo sa Liberal Arts sa AB?

Abulad: Hindi. Nagshift ako sa seminaryo muna [laughs].

De Leon: Ah, pumasok po pala talaga kayo sa seminaryo? Dito po sa seminaryo natin sa UST?

Abulad: Hindi, hindi. Sa mga Jesuits. Dahil ang nanay ko, may estudyante siyang naging Obispo na doon nag-aral sa San Jose Seminary - hindi doon sa seminaryo kung saan [nag-aaral] para maging Jesuits. Dinala ako ng Obispong iyon doon sa San Jose Seminary. I stayed there for just one year [laughs]. Tapos lumabas ako.

De Leon: Philosophy na po ang kinuha ninyo pagkalabas ng seminaryo?

Abulad: Noong sabihin ko sa aking Rector na lalabas ako, ang tanong niya sa akin ay kung anong kukunin ko paglabas? [laughs] Nakatunganga ako sa kanya at hindi ko alam ang sasabihin. At, alam ko sa sarili ko na hindi ako babalik sa Science. Noong makita sigurong hindi ako nagsasalita, siya na rin ang nagsabi sa akin: "Do philosophy and later on you will come back!" So, sabi ko, "Opo!" Masunurin akong bata eh. So, bumalik ako sa University of Santo Tomas at diretso ako sa philosophy.

De Leon: So, parang aksidente lang po pala na napunta kayo sa philosophy dahil lamang sa pagsunod ninyo sa paghimok ng isang Rector [laughs].

(C) 2016 Emmanuel C. De Leon

http://www.kritike.org/journal/issue 18/de leon june2016.pdf

ISSN 1908-7330

(c) $)$ BY-NC-ND 
Abulad: Oo [laughs]. Kaya sabi ko, "I'm not following my head, I'm following my intuition." [laughs] Hanggang ngayon, iyon pa rin ang sinasabi ko, "I'm following my intuition." And you know why? Kasi ang pinakamababa kong grade noong nasa College of Science ako ay Logic at Ethics. 'Yung dalawa ang pinakamababa kong grades, 81 yata [laughs]. Pero, noong sabihin sa akin ng pari [ng San Jose Seminary] na pumasok ako sa philosophy, pasok ako ng philosophy. At, doon naman ako nag-enjoy sa philosophy. Especially noong second semester ng third year, kasi tapos ko na ang second year eh, dumating naman si Dr. Quito. At nabago ang philosophy sa UST [laughs]. Tuwang tuwa yung mga kaklase ko [laughs].

De Leon: Bago po dumating si Dr. Quito galing sa Switzerland, ano po ba yung sistema ng pilosopiya na mayroon sa UST?

Abulad: Eh kaya nga siguro ako naging 81 [sa philosophy noong nasa College of Science ako] [laughing out loud]. Pero, I consider myself as a good studentmasunurin at ginagawa ko kung anong sabihin ng teacher-kaya natuto naman ako ng Thomistic philosophy. Talagang solid ang aking Thomistic background.

\section{The Awakening from Dogmatic Slumber}

De Leon: Noong dumating po si Dr. Quito sa UST, ano po kaagad ang itinuro niya sa inyo?

Abulad: Basta ang alam ko, suyang-suya na ang mga kaklase ko sa Scholastic philosophy. 'Yun ang maaari mong tawaging "sick and tired." So, noong dumating si Dr. Quito, parang fresh air [laughs]. Hindi lang naman si Dr. Quito ang dumating. Sa Ateneo, dumating sina Dr. Reyes, kaya phenomenology at existentialism ang naging foundation ng Ateneo. Tayo dito, Dr. Quito almost singlehandedly taught phenomenology at existentialism. Kaagad naging successful ang phenomenology at existentialism [dito sa UST]. And more than that, what I really like about Dr. Quito, almost every semester, may ibinibigay siya parating bagong philosophy sa amin. Noong matapos ako ng Bachelor of Philosophy, immediately pumunta ako sa Graduate School [ng UST]. At, lagi akong nageenrol kay Dr. Quito. Every semester, may bago kaming [nakukuhang] philosophy sa kanya. Sa kanya ko nakuha ang structuralism, hermeneutics, Marcuse, Marx, Plotinus, at pati Oriental philosophy siya rin ang nagbigay sa amin. Name it! At sa kanya lahat iyan nanggaling.

(c) 2016 Emmanuel C. De Leon

http://www.kritike.org/journal/issue 18/de leon june2016.pdf

ISSN 1908-7330

(cc) BY-NC-ND 


\section{INTERVIEW WITH ROMUALDO ABULAD}

De Leon: Itinuturing po ang mga nasabi ninyong kurso na "bago" noong panahon ninyo dito sa UST? Fresh air po talaga?

Abulad: Fresh air talaga ito! At, dahil fresh air nga, 'yung stable system dito ay medyo nayanig. Ang lahat kasi noon ay halos Tomista eh [laughs]. Solo si Dr. Quito na dumating at hindi naman nila magawang ma-ignore. So, "hero" itong si Dr. Quito noong panahong iyon.

De Leon: Pero, si Dr. Quito po, matatag din ang kanyang pundasyon pagdating sa sistemang Aristoteliko-Tomistiko.

Abulad: Kaya hindi siya mabasta-basta ng kahit na sino diyan. Sinong mga nandiyan [noong panahong iyon]? Sina Antonio Piñon, Salvador Gonzales, Ariston Estrada, mga diehard ang mga yan at mga tried and tested.

De Leon: 'Yan po ba yung tinatawag na "be-all and end-all" pagdating sa Tomismo?

Abulad: 'Yan! Right! Noon ay lahat dapat nasa textbook 'yan eh. Kapag nagkamali $\mathrm{ka}$ at hindi alinsunod sa pilosopiya ni Santo Tomas, parang pakiramdam mo ay malaki ang pagkakasala mo. Mortal sin! [laughs]. Mapupunta ka sa impyerno. But, with Dr. Quito? The new philosophies came.

De Leon: Pero, mukha pong sa nabasa ko mula sa mga isinulat ninyong artikulo, hindi nyo naman po minamasama ang Tomismo, tama po ba?

Abulad: Ah, hindi.

De Leon: Sa inyo pong artikulong may pamagat na "Contemporary Filipino Philosophy" (Karunungan 5, 1998), nabanggit po ninyo ang dalawang mukha ng Tomismo sa Pilipinas. Ipinaliwanag po ninyo na mayroong mabuti at masamang naidulot ang tradisyon ng pamimilosopiyang matatawag na Tomismo. Wika po ninyo,

Thomism in this country became indomitably stubborn that it started giving the impression that no truth could possibly lie outside of its pre-established framework. In my youth, I saw very clearly how intellectual doggedness could prove fatal to an aging philosophy (3).

Sa sumunod naman pong pahina, sinabi ninyo,

(C) 2016 Emmanuel C. De Leon

http://www.kritike.org/journal/issue 18/de leon june2016.pdf

ISSN 1908-7330

(c) ) BY-NC-ND 
Indeed, one incontestable virtue of a solid background in the system of St. Thomas lies in its formative value. By "formative value" I refer to the fact that student who has an early exposure to Thomism tends to develop a wellorganized and lucidly logical manner of thinking, which, to use Kant's words, constitutes a conditio sine qua non for a competent philosophy (4).

Maaari po bang ipaliwanag pa, ano po bang nakikita ninyong positibo sa sistema ng Tomismo?

Abulad: Oo. Ganun nga. Kung hindi dumating si Dr. Quito, talagang suyangsuya na ang aking classmates [laughs].

De Leon: 'Yun ang term ano po, "suyang-suya." [laughs]

Abulad: Oo. 'Yung para bang sa tuwinang kakain ka ay pare-pareho ang ulam mo. Eh kahit na masarap ang ulam mo, kapag sobra naman, [nakakasuya rin]. Tapos, in the end, sasabihin pa sa 'yo na hindi tama itong sinasabi mo. Parang naging objective tuloy masyado-right or wrong. At wala ka nang kalayaang mag-isip. Especially, you are young [during that time]. Bakit ganyan ang sitwasyon, eh philosophy 'yan? You can see what kind of trap it could be, ano? Hindi lang 'yan sa Tomismo maaaring mangyari. Analytic philosophy could be like that too. Kahit na ano. Marxism could be like that also. Lahat na sistema ay maaaring maging dogmatic, na parang sasabihin mo na ito lamang ang totoo at lahat ng labas dito ay mali. Ah, hindi na 'yan uubra ngayon.

De Leon: Bukod po doon sa sinasabi ninyong tulong ng Tomismo sa pagiging sistematikong mag-isip, ano pa pong nakikita ninyong positibo dito?

Abulad: Una, hanggang ngayon, naniniwala pa rin ako na dapat unahin ang Aristotelico-Thomistic philosophy. At, naniniwala pa rin ako na iyan ay magandang pundasyon. Pero, sabi ko nga, hindi ka dapat manatili na lamang diyan. I-establish mo lang 'yan, at pagkatapos, lampasan mo 'yan. Alam mo, may nakikita pa rin akong hindi makalampas doon hanggang ngayon.

Ang hirap kapag ikaw ay naging Tomista, you take everything said by St. Thomas hook, line, and sinker [laughs]. Si Santo Tomas ay hindi naman ganyan. Pinag-aaralan niya ang mga sciences ng kanyang panahon. Kaya nga nakacompose siya ng Summa Theologiae-lahat ng mga 'yun ay galing sa mga sciences na available noong panahon niya. Kaya nga, open-minded si St.

(c) 2016 Emmanuel C. De Leon http://www.kritike.org/journal/issue 18/de leon june2016.pdf

ISSN 1908-7330 
Thomas. In fact, pwede mong sabihin na rebolusyonaryo siya-intellectual revolutionary siya-noong panahon niya. Tapos, ipepreso natin siya ng ganun?

Pangalawa, hindi tinapos ni Santo Tomas ang kanyang Summa at ayaw niyang tapusin kahit kaya naman niyang gawin. Bakit? Sabi niya, "Lahat 'yan basura!" 'Yan ang paborito kong St. Thomas Aquinas. Hindi siya dogmatic na pilosoper. Pero, pwede mo siyang gawing dogmatic. Pero, hindi siya naging dogmatic. At ang nagsalba sa kanya sa pagiging dogmatic ay 'yun nga na ayaw niya [na tapusin ang kanyang Summa]. He could have, but he did not finish his Summa Theologiae, leaving a lot of doors open for possibilities.

Pangatlo, ngayon may encyclical si Pope Francis, itong Laudato Si'. Ang inirerekomenda niya ay itong "integral ecology." Ano itong integral ecology na ito? It's about time na magkasama-sama ang mga disciplines. Nandiyan ang environmental ecology, social and political ecology, at nandiyan din ang ethical ecology. Lahat ay dapat sama-sama iyan. Para sa akin, si Pope Francis ay isang postmodern na Santo Tomas at hindi medieval. Sabi ko nga, "integral ecology" is another name for philosophy. Hindi siguro conscious si Pope Francis doon. Pero, integral ecology is another name for philosophy. Philosophy is integral ecology. And the best example is still St. Thomas Aquinas. Summa Theologiae is the summary of all knowledge, which is not just a philosophy but also a theology.

Makikita mo ang value ni Santo Tomas h'wag mo lang ipipilit na "everything is made up of matter and form." At, kung ipipilit mo yan, naku po! Ngayon ang ibig sabihin ng "matter" ay ibang iba na kesa noong panahon ni Aristotle o ni Santo Tomas. "Form?" Naku, mahihilo ka sa napakaraming kahulugan. Kaya, kung magsulat ka at itratranslate mo sa ating lengwahe, o kung paano natin naintindihan 'yan, ibang-iba na ang theory of hylemorphism.

\section{Teaching Philosophy}

De Leon: Nagtapos po kayo sa UST nang 1969. Tama po?

Abulad: Tama.

De Leon: Tapos, kaagad din kayong nagturo sa UST ...

Abulad: Kinuha agad ako.

De Leon: Opo. Maaari po ba ninyong ibahagi kung sinong naghikayat sa inyong pumasok sa pagtuturo ng pilosopiya dito sa UST?

(C) 2016 Emmanuel C. De Leon

http://www.kritike.org/journal/issue 18/de leon june2016.pdf

ISSN 1908-7330

(c) $)$ BY-NC-ND 
Abulad: Gusto ko talagang magturo. Intuition ang aking sinusunod. It comes from me, pero si Dr. Quito yung nag-suggest na "mag-apply ka!" Nag-apply naman ako at magagaling din yung mga kasabay kong nag-apply. Kilala ko na magagaling din sila. Pero, ako ang tinanggap [laughs].

De Leon: Ito na po 'yung panahon ni Presidente Marcos di po ba?

\section{Abulad: Oo.}

De Leon: Anu-ano pong uring pakikibaka ang masasabi ninyo na ginawa ng mga Tomasino noong panahong iyon?

Abulad: Oo, oo. Mararamdaman mong wala kang silbi kung nagtuturo ka lamang sa classroom. Na nagtuturo ka pa ring tulad ng dati at parang walang pakialam. Kapag ganoon, mararamdaman mong walang kabuluhan ang iyong ginagawa.

Kasama kami! Kasama kami! Lumalabas kami ng unibersidad [para makibaka]. Siguro ang administration ay medyo nag-iingat kaya medyo konserbatibo ang tingin. Pero, ang faculty at estudyante ay hindi ganoon. From day one of Martial Law, ayaw na namin [dito]. Nakikita naming hindi ito tama.

De Leon: And then, mas pinili po ninyong mag-Masterado sa Ateneo?

Abulad: Ah, kasi ang aking pilosoper ay si Kant. Nagsimula ako dito sa UST, of course narinig ko na [ang turo ni] Dr. Quito. Dahil sa kanya kaya ako naimpluwensiyahang mag-Kant. Sa klase namin sa undergrad hanggang Graduate School, laging sasabihin sa amin ni Dr. Quito, "Kapag wala kang Kant, may kulang sa iyong edukasyon." Ang sitwasyon, dahil palagay ko naman simula noong pumasok ako ng philosophy ay nag-aaral na akong mabuti; naintindihan ko naman yata ang mga itinuturo sa akin; at binigyan ni Dr. Quito ng pinakamahabang panahon ang pagtuturo kay Kant. And yet, sa lahat ng pinag-aralan ko ang pinakamalabo ay si Kant. Hindi ko siya maintindihan. "I Kant understand." [laughs].

De Leon: Paano po nangyari yun? Iniisip ko pong parang imposible yun sa isang Br. Abulad [laughs].

Abulad: Eh naintindihan ko si Hegel, pero hindi ko talaga maintindihan si Kant kahit binigyan ni Dr. Quito ng pinakamahabang panahon ang pag-aaral namin kay Kant. Saka, isiniksik sa amin na kapag wala kang Kant ay kulang

(c) 2016 Emmanuel C. De Leon

http://www.kritike.org/journal/issue 18/de leon june2016.pdf

ISSN 1908-7330

(cc) BY-NC-ND 
o may lacuna o gap ang iyong edukasyon. So, pinilit kong pag-aralan si Kant. After a while, narealize kong wala na akong matutunan tungkol kay Kant sa UST. At iyon ang nag-move sa akin papuntang Ateneo. Akala ko [laughs], mayroong magtuturo sa akin tungkol kay Kant doon. So, nag-enrol ako doon, especially kay Dr. Ramon Reyes, dahil siya ang supposedly Kant expert doon sa Ateneo. Pero, in the end, wala. Ako pa rin ang mag-aaral kay Kant-on my own. And it took me something like ten years bago ko nasabing kahit paano ay may nakuha na akong linaw kay Kant.

De Leon: Mayroon po bang malinaw na pagkakaiba sa sistema ng pamimilosopiya sa Ateneo at UST noong mga panahon na iyon?

Abulad: Siguro, hindi ako fair dito, pero dahil galing ako kay Dr. Quito eh. Then, pagdating ko sa Ateneo, walang makacompare sa kanya [laughs].

De Leon: Wala pa po ba noon sina Roque Ferriols at iba pang philosophers nila doon?

Abulad: Naroon! Naroon na silang lahat. 'Yun na nga eh. Nandoon na sina Roque Ferriols - siya nga ang aking naging adviser sa aking thesis.

De Leon: Ganun po pala. Ano po ang sinulat ninyong thesis noon?

Abulad: Tungkol kay Kant din. Pinagcompare ko si Kant at si Shankara.

De Leon: Iyan din po yata ang inyong sinulat na disertasyon?

Abulad: 'Yan nga ang aking disertasyon. Teka, sa Ateneo pala ang sinulat kong thesis ay tungkol sa epistemology at metaphysics ni Kant. At hanggang ngayon ay alam ko kung anong mali sa trabaho ko [laughs]. Pero, hindi nila napansin. Kita mo, hindi nila alam [laughs]. Pero, ako alam ko na ngayon, may mali talaga [laughs].

De Leon: So, andun na po pala noon sina Roque Ferriols.

Abulad: Sina Roque Ferriols, [Manuel] Manny Dy [Jr.], at [Leovino] Leo Garcia - they were already there.

De Leon: Umupo din po kayo sa klase ni Ferriols?

Abulad: Oo. Pero, Ingles ang language niya sa klase namin.

(C) 2016 Emmanuel C. De Leon

http://www.kritike.org/journal/issue 18/de leon june2016.pdf

ISSN 1908-7330

(c) BY-NC-ND 
De Leon: Hindi pa po siya nagsisimulang magturo sa Filipino?

Abulad: Nagtuturo na siya sa Filipino, pero ang partikular naming klase ay Ingles ang gamit.

\section{Further Studies}

De Leon: Natapos naman po ninyo ang inyong Doktorado sa pilosopiya sa ating alma mater na UST noong 1978.

Abulad: Oo. Kasi walang ibang unibersidad sa Pilipinas ang nag-ooffer ng Ph.D. sa philosophy kundi ang ating unibersidad noong mga panahong iyon.

De Leon: Opo. Tapos, isang taon po ang makalipas, nagpunta naman kayo sa Alemanya, sa Unibersidad ng Hamburg (1979-1981) upang mag-aral pa lalo ng pilosopiya. Maaari po ba ninyong isalaysay ang inyong mga karanasan at aral na natutunan sa ginawa ninyong ito?

Abulad: Kasi pagkatapos ko ng aking Ph.D. dito, alam ko dahil intuition nga ang aking sinusunod, hindi naman utak, hindi ako smart [laughs], kaya intuition ang aking sinusunod; alam ko na ang kulang ko. Hindi pa ako kuntento sa aking Kant. And the reason really is I had not read him in the original German text. Nag-aaral na rin naman ako ng German language sa Goethe Institute, pero hindi ko pa noon nabasa si Kant sa original German. So, alam ko na ang dapat kong gawin ay pumunta ng Germany para basahin si Kant sa wikang Aleman. And you know, sinusunod ko lang naman ang intuition ko, nag-apply ako sa Alexander von Humbolt Foundation. Ang bilis-bilis. Sumulat ako sa mga universities at maraming professors ang sumagot sa akin. Sayang nga lang, wala akong kamuwang-muwang. Sabi ko, "ito yata ang mas madali." [laughs] Kaya yun ang pinili ko. Next time alam ko na [laughs].

De Leon: Tatlong taon po kayo doon?

Abulad: Dalawa.

De Leon: Ito po yun panahon na nakatayo pa ang Berlin Wall di po ba?

Abulad: Oo. Kaya nga hindi ako nakapunta sa Königsberg dahil sakop yan ng Russia, ng mga komunista.

De Leon: Talaga pong na-in love po kayo kay Kant, ano po?

(c) 2016 Emmanuel C. De Leon

http://www.kritike.org/journal/issue 18/de leon june2016.pdf

ISSN 1908-7330

(cc) BY-NC-ND 
Abulad: I don't know if that is "in love," but I have no regrets. Basta ang nagtulak sa akin kay Kant ay hindi ko siya maintindihan [laughs]. At 'yung mga naging guro ko, hindi naman nakatulong sa akin. I hope they could teach me more, pero hanggang doon lamang yata ang alam nila, ano? Kaya, sa katupus-tapusan, ako talaga ang nag-aral ng Kant.

De Leon: Sino pong pilosoper sa Germany ang nakadaupang-palad ninyo doon?

Abulad: 'Yung adviser ko. Well, kung post-doctoral 'yan, magkasama lang kayo ng ... you don't even call it mentor ... pereho kayo eh, equal ang treatment sa iyo. Sabi ko nga sa kanya, gusto ko yatang mag-doctorate dito. Sabi niya, "that's unheard of." [laughs] May "Doctor" ka na [laughs].

De Leon: Pagkatapos po ay lumipat kayo ng De La Salle College?

Abulad: All this time, nasa De La Salle na ako. Dito sa UST ay nagturo lamang ako ng mga dalawa o tatlong taon. Tapos, si Dr. Quito kasi ay na-pirate ng De La Salle [laughs]. Eh noon naman ay kaigtingan nga ng mga social movements dito. Kaya, maligaya na rin ako noong sabihin sa akin ni Dr. Quito na doon na lang din ako sa De La Salle. At, hindi na rin ako komportable sa atmosphere ng university, mas komportable ako sa mas "rebolusyonaryo." [laughs]

De Leon: Sino po ang mga kasama ninyo sa departamento ng pilosopiya sa De La Salle noon? Ano pong tradisyong pilosopikal ang mayroon noon sa La Salle?

Abulad: Well, sa departamento, si Dr. Quito nga ang number one.

De Leon: Maituturing po ba ninyo siyang founder ng philosophy doon?

Abulad: Siya ang paradigm shift. Noong dumating siya, nabago lahat. Siya halos ang nagturo ng lahat sa amin, pero hindi ako nasusuya. Malayong malayo sa kanya ‘yung mga kasabayan niyang nagturo ng pilosopiya. I don't want to name names anymore. But, they are good humans. Pero, malayo ang agwat ni Dr. Quito sa kanila. Kaya hindi ako masyadong nag-enjoy halimbawa sa rational psychology ko. Sa aesthetics, may magaling akong teacher, kaya lang entertaining lang siya sa klase at saka maganda siya. Kaya ayaw ko ng pareport-report na style sa pagtuturo, wala akong natututunan sa ganyang style except 'yung aking inireport na topic. Kaya sa aesthetics,

(c) 2016 Emmanuel C. De Leon

http://www.kritike.org/journal/issue 18/de leon june2016.pdf

ISSN 1908-7330

(c) BY-NC-ND 
pinareport ako tungkol kay Rembrandt, hanggang ngayon alam ko pa ang Rembrandt. Pero, 'yung iba ay hindi ko na alam. Oo, maganda rin nga 'yung aking teacher sa aesthetics noon [laughs].

De Leon: Kung pagbabatayan po ang dami ng inyong sinulat kasama si Dr. Quito, talagang masasabi po na napakalaking inpluwensiya niya sa inyo.

Abulad: Sure. Kasi siya ang namamahala nga ng departamento namin sa De La Salle. Very very active at very creative si Dr. Quito. Silang dalawa ni Br. Andrew Gonzales ang magka-tandem. At 'yang si Br. Andrew Gonzales ay genius pagdating sa school administration. Kay Br. Andrew nakaranas ako ng mahusay na pamumuno sa eskwelahan, that's really great leadership. At, magka-tandem sila ni Dr. Quito na napakahusay din pagdating sa departmento. Kaya, maganda talaga ang tandem nila. At sa palagay ko ang magandang ginawa ni Dr. Quito sa De La Salle ay yung pagsisimula ng kultura ng pagsusulat. Nagkasundo sila ni Br. Andrew sa larangan na 'yan. Kaya nahikayat din akong magsulat. Kung pababayaan mo lang ako, baka hindi ako nagsulat. Halos lahat ay hikayat niya. Dahil sa hikayat niya kaya nagsulat kami ng ensayklopediya, ng libro sa pilosopiya ng tao, itong mga textbooks na ito na magaganda, si Dr. Quito ay may ideya ng mga 'yan.

De Leon: At hindi lang po siguro sa pagsusulat, noong panahon po ninyo, may kultura ng pagsusulat gamit ang wikang pambansa.

Abulad: Kasama na 'yan. Si Dr. Quito ang alam kong kauna-unahang pilosopong Pilipino na nagsulat sa Filipino. Una pa siya kesa kay Roque Ferriols.

\section{The Use of Filipino Language}

De Leon: Napansin ko po na marami-rami talaga kayo nasulat gamit ang wikang pambansa. Kasama kayo sa nagsulat ng napakahalagang Ensayklopediya ng Pilosopiya (1993), andiyan din ang Ang Pilosopiya ng Tao (1980) na sinulat ninyo kasama si Dr. Quito, at mga artikulong tulad ng "Bayani," "Ang Diwa at Kaisipan ng Pilipino sa Kasaysayan ng Himagsikan," "Kalooban Mo, Kagustuhan Ko," "Mga Puna Tungo sa Pagasa," "Pilipino sa Pilosopiya," "Ang Pilosopiyang Pranses sa Pananaw ng Isang Pilipino," "Ang Pilosopiya ng Salapi ni Georg Simmel," "Si Kant at ang Pilosopiya sa Pilipinas," “Ang Teorya ng Ebolusyon," “Ulat Bunga ng Pakikipamuhay," at kasama rin kayo sa artikulong "Ulat Tungkol sa Sanggunian at Leksikon ng Pilosopiya." Siguro po ay mayroon pa akong nakaligtaang banggitin.

(c) 2016 Emmanuel C. De Leon

http://www.kritike.org/journal/issue 18/de leon june2016.pdf

ISSN 1908-7330 


\section{INTERVIEW WITH ROMUALDO ABULAD}

Abulad: Oo. Meron pa, meron pa.

De Leon: Maaari po ba ninyong ilahad kung paano kayo nagsimulang magsulat ng mga pilosopikal na akda gamit ang wikang pambansa?

Abulad: Kasi unang-una ay Tagalog ako. Pagkatapos, marunong akong masulat. At syempre ang susulatin ko ay pilosopiya. At saka si Dr. Quito ang halimbawa ko. Si Dr. Quito ay Kapampangan, kaya ang Tagalog niya ay Kapampangan din [laughs]. Pero, nagsumikap siyang magsulat sa Filipino kahit na ang Tagalog niya ay hindi mo masasabing polished Tagalog. Nagpilit siyang magsulat sa Filipino dahil naniniwala siyang dapat nating linangin ang sariling atin. Galing siya sa mga bansa na ang ginagamit ay sariling wika sa pagtuturo at pagsusulat. Dito sa atin, medyo tayo ... Ano bang term doon?

\section{De Leon: Kolonyal?}

Abulad: Kasama na rin 'yun, may pagka-kolonyal tayo. Look around. Ang Vietnam ay sa Vietnamese nagtuturo at nagsusulat, ang mga Japanese sa Japanese, ang Chinese sa Chinese, ang Indonesian sa Indonesian. Pero tayong mga Pilipino sa Ingles [laughs]. Kaya lang, no turning back na. Mahihirapan tayo. Pero, baka wala na ako dito, at ikaw din wala na dito, bago talagang masabi na tayo ay nagpipilosopiya sa Tagalog. At isa pang problema diyan ay ang Tagalog naman ay isa lamang sa mga wika natin dito sa Pilipinas.

De Leon: May rehiyunalismo po ba kayong napapansin?

Abulad: Oo. Bakit hindi Cebuano? Bakit hindi Ilokano? Bakit hindi Kapampangan? May karapatan din sila.

De Leon: Pero, ang sinasabi po nilang wikang Filipino ay pinagsama-samang lengwahe sa Pilipinas.

Abulad: Eh nasaan 'yun? Wala pa talaga. 'Yung "Filipino" na iyon, wala pa 'yun eh.

De Leon: Nasa diksyunaryo pa lamang po yata [laughs].

Abulad: Oo.

(C) 2016 Emmanuel C. De Leon

http://www.kritike.org/journal/issue 18/de leon june2016.pdf

ISSN 1908-7330

(c) BY-NC-ND 
De Leon: Nasabi po ni Dr. Alfredo Co sa kanyang artikulong "Doing Philosophy in the Philippines Fifty Years Ago and Fifty Years From Now," na marami na ang pinanghinaan sa paggamit ng wikang Filipino sa gawaing pamimilosopiya. Tama po ba ang sinasabi ni Dr. Co? Kung tama po, isa po ba kayo sa masasabing "pinanghinaan na ng loob"?

Abulad: Hindi naman. Season season lang 'yan eh [laughs]. Isa pa, talagang hindi natin kayang labanan ang Ingles. Kung Tagalog lang ang ating gagamitin sa pag-aaral, itong alam natin sa pilosopiya ay hindi natin makakamit. Isa tayo sa pinakamaulad sa pilosopiya dito sa Asya because of English. Kung gagamitin natin ay Filipino "lang," anong access natin sa mga primary works?

De Leon: Mahalaga ang parehong wika para sa inyo?

Abulad: Oo. Ang mahalaga ngayon, ang mga nag-aaral ng pilosopiya ay kailangang magaling sa Ingles para may access sila sa mga translations. So, I have nothing against translations. At, itong mga magagaling na ito sa Ingles at saka sa pilosopiya, ito rin ang magagaling sa Tagalog. Sana ay magkatuwang sa kanila 'yung pagsusulat din sa Tagalog, pamimilosopiya rin sa Tagalog. 'Pag ganyan, dahan-dahan 'yang lalago; magkasabay na lalago ang Tagalog at Ingles.

De Leon: Hindi po ba napapag-iwanan ang Tagalog sa takbo ng pamimilosopiya sa ating bansa?

Abulad: Hindi lang naman sa pilosopiya. Mabuti pa nga at nagtatagalog tayo [laughs]. Talagang Ingles dito, kaya nga may mga foreign students tayo. Kung wala tayong Ingles baka wala ang mga 'yan. Ang problema diyan ay ang klase ng ating pagtuturo at pagtatalakay ng pilosopiya-'yung uri ng ating edukasyon. Hindi 'yan question ng language sa akin.

De Leon: Ano pong uri ng edukasyon ang inyong kinakalaban?

Abulad: Wala naman akong nilalabanan [laughs]. Mabuti nga ngayon hindi na ako nagagalit. Natatandaan ko, two years ago, "Ano? Gusto ninyo na puro na lang quizzes? Pambata 'yan! Pang-High School yang mga quizzes, graded recitation, True or False." 'Yang mga 'yan [ang ayaw ko]. Sabi ko sa mga estudyante ko, "I will treat you as adults. Hindi ko igregrade ang inyong recitation. And be open. You don't have to be afraid. Kung may ayaw kayo sa sinabi ko, sabihin ninyo at mag-usap tayo." 'Yung ganun ba-mag-usap. Kaya lang, baka sabihin naman na extreme ako. Kasi ayaw ko ng reporting sa 
klase, dahil inaasahan mo ang guro na magturo. Pero, hindi naman nangangahulugan na sa lahat ng pagkakataon ay hindi bagay ang reporting. Istratehiya 'yan ng pagtuturo eh. Kailangang creative ka. Kaya nga lang sa sistema ng edukasyon natin, nagtuturo tayo 90\% dahil sa sweldo [laughs].

De Leon: Nagpapublish para lamang sa promotion [laughs].

Abulad: 'Yan, tama yan.

De Leon: Br. Romy, gusto ko pong balikan 'yung mga unang taon ninyo ng pagtuturo dito sa UST noong 1969. Sinu-sino po ang mga nakasama ninyo sa departamento ng pilosopiya noon? May departamento na po ba ng pilosopiya noon?

Abulad: Meron.

De Leon: Pwede po ninyong idescribe kung paano ito noon?

Abulad: Sandali ha! Kasi ang palagi ko lamang nakikita noon ay si Dr. Quito [laughs]. Pero, iisa ang aming Faculty Room, dito sa $2^{\text {nd }}$ floor [ng St. Raymund's Building]. Katabi 'yan ng opisina ng Dean. Lahat kami nandoon-literature, economics, lahat ng subjects; iisa ang Faculty Room namin. Parang ang Department of Philosophy noon ay si Dr. Quito lamang ang natatandaan ko [laughs].

De Leon: Batang-bata po kayo noon. Ano pong istilo ninyo sa pagtuturo?

Abulad: Ewan ko. Pero, parang nasa dugo ko talaga ang pagtuturo. At, galing ako kay Dr. Quito at nakita ko kung papaano siya magturo. At, mayroon din akong lousy teachers na sabi ko sa sarili ko, "Hindi ko tutularan ang mga ito." [laughs]. Kaya, sana naman, kahit na noong simula pa lamang ay pinilit kong ayusin ang pagtuturo. At habang nagtuturo ako, wala sa isip ko 'yung kwarta. In fact, ngayon ko lang nakikita na mahirap pala ako noong panahong iyon [laughs].

De Leon: Gumamit din po ba kayo ng wikang Filipino sa pagtuturo?

Abulad: Hindi. Parang hindi uso talaga.

De Leon: Ano pong dahilan? Dahil po ba walang mga tekstong gagamitin?

(C) 2016 Emmanuel C. De Leon

http://www.kritike.org/journal/issue 18/de leon june2016.pdf

ISSN 1908-7330

(c) BY-NC-ND 
Abulad: Isa na 'yun. Pero, dahil din noong panahon ko nagsimula na rin 'yung ayaw nila 'yung paggamit ng TagLish. Gusto nila 'yung Bilingual. Ang ibig sabihin ng bilingual noon, kung Tagalog ang gagamitin mo dapat Tagalog all the way; at kung Ingles ang gagamitin mo, Ingles all the way. And the reasoning is kapag pinagsama mo sila, TagLish ang ginawa mo, chopsuey, baluktot pareho.

De Leon: So, mas pinili niyo pong gamitin ang wikang Ingles?

Abulad: Walang choice ba. Pero, kung sabihin sa akin na Tagalog ang pagtuturo ay walang problema sa akin. Kaya ko [laughs].

De Leon: Mayroon po kayong sinulat na librong Introduction to Philosophy (2001) kasama si Dr. Ceniza?

Abulad: Yeah!

De Leon: Ano pong nag-udyok sa inyong gawin 'yun? May pangangailangan po ba noong mga panahon na 'yun?

Abulad: Actually, dito 'yun [sa UST] nanggaling. Si Dr. Co ang nagbigay sa amin ng proyekto na 'yan - two-volume work. Isinama ko lang si Dr. Ceniza dahil parang alam namin na he will be going soon-parang may ganoon na kaming premonition sa kanya. 'Yung first volume ay tungkol sa Cosmology, Metaphysics, at ....

De Leon: Theodicy?

Abulad: Yeah, Theodicy. 'Yung Cosmology part, kinausap ko si Dr. Ceniza [na siya ang magsulat]. Good enough, 'yun ang last work niya. 'Yung second volume naman, ibinigay ko rin ang aking ginawa doon. Kaya lang nagkagulo sila ata doon [laughs].

De Leon: Ano naman pong contents noong second volume?

Abulad: Psychology, Ethics, and Epistemology. Those three. Tapos na 'yun, naayos ko na 'yan. Isinubmit ko na. Tapos nagkaroon ng politika diyan. Kaya sabi ko, inyo na lang 'yan. May politika na kasi [laughs].

(c) 2016 Emmanuel C. De Leon http://www.kritike.org/journal/issue 18/de leon june2016.pdf

ISSN 1908-7330 


\section{UST Philosophy Department Before and Today}

De Leon: Mga panghuli na po [laughs]. Ah, kung inyo pong paghahambingin ang istilo ng pamimilosopiya noon at ngayon, kasama na siguro ang hilig ng mga estudyante sa pilosopiya noon at ngayon, ano po ang inyong napapansin? Mayroon po bang pagkakatulad at pagkakaiba?

Abulad: I will not go back. Ang aking isip ay palaging paabante, hindi paatras. Kaya, ang isang hindi ko babalikan ay 'yung walang ibang pilosopiya sa UST kundi Tomismo at Iskolastisismo. Hindi na ako babalik diyan. Pero, sisiguraduhin din dapat na malakas ang pundasyon natin sa pilosopiyang Iskolastiko at maging sa Tomismo. Pundasyon ang kursong ‘yan.

Alam mo noong nandoon ako sa Aparri [sa conference ng Philosophical Association of the Phillippines], just two weeks ago, nakinig ako ng ibang parallel sessions. Napuna ko magagaling 'yung mga batang faculty sa UST. At, maganda ang kanilang philosophical attitude. I think we are succeeding in developing our young faculty here. Balanse sila. Alam nila [ang kanilang pundasyon], pero hindi sila sarado. Hindi sila trapped. Postmodern sila [laughs].

De Leon: Ano pong nakikita ninyong challenges pa sa philosophy sa buong Pilipinas, in general, at sa paraan ng pamimilosopiya natin dito sa Unibersidad ng Santo Tomas?

Abulad: Alam mo, dapat ipakita natin sa bansa na mahalaga ang pilosopiya. Kailangang makita ng bansa ang kahalagahan nitong ating ginagawa. Kaya tuwang-tuwa ako noong ipalabas ni Pope Francis 'yung kanyang Laudato Si'. Hindi niya lang siguro alam na ang sinasabi niya doon ay "Go back to philosophy!" 'Yung kanyang [ideya] ng "integral ecology," pilosopiya 'yan. That's all over again Hegel, for example. That's all over again St. Thomas Aquinas. That's all over again Plato and Aristotle. That's all over again all philosophy na walang closure-na bukas ang pag-iisip. 'Yung postmodern thinking, very useful, very practical, very helpful in the currest thrust of society. Towards what Kant called "eternal peace." [laughs] Philosophy 'yan! At dapat makita 'yan ng ating lipunan. 'Yun sana ang sama-sama nating maging main project.

Sama-sama nating gagawin. Hindi tayo dapat mag-away-away [laughs]. Kasi ang pilosopiya, conversation 'yan eh. Bakit nakarating hanggang dito ang Western philosophy? Dahil walang tigil ang pag-iisip nila. At hindi lang isang tao ang nag-iisip. Lahat sila nag-iisip. Tulong tulong sila.

(c) 2016 Emmanuel C. De Leon

http://www.kritike.org/journal/issue 18/de leon june2016.pdf

ISSN 1908-7330

(cc) BY-NC-ND 
Nag-aaway-away pero, sa katapus-tapusan, pinapatakbo nila ang kaalaman. Ganyan din ang dapat mangyari sa Filipino philosophy.

De Leon: Sa palagay po ninyo wala pa niyang mayamang kultura ng matinong pag-aaway-away?

Abulad: Ah, medyo lumalakas na. Medyo nararamdaman ko na nagkakaroon na. So, I'm not worried about the future of philosophy. Kaya lang, ang dami-daming pumupunta sa psychology. Akala ng mga tao, psychology will save the world [laughs]. No, it's philosophy that will change the world.

De Leon: Ano po bang advantage kung talagang may grasp ka sa philosophy bilang tao?

Abulad: Yeah! Well, on the higher level, walang magaling in any discipline na walang pilosopiya. The best artist has a philosophy, the best scientist has a philosophy.

De Leon: Tama po. Nabasa ko po na talagang sinabi ni Albert Einstein na kung ano siya ay dahil sa kanilang inaral na pilosopiya mula sa pagkabata. Hindi nila kinakalimutan ang inaral nilang pilosopiya simula elementary hanggang sa pagtanda nila.

Abulad: Yeah! If you are good in your discipline, you will eventually be doing the philosophy of it. Sa buhay, ganoon din. You are as good or as bad as your philosophy. Basahin mo si Marx, ang sinusulat niya ay tungkol sa economics pero isa siyang pilosoper; Si Amartya Sen ....ang galing galing ni Amartya Sen, isa rin siyang pilosoper.

De Leon: Ano naman po ang inyong "huling habilin?" Hindi naman po pala "habilin," ano pong inyong mga hamon sa mga batang namimilosopiya ngayon sa Pilipinas?

Abulad: Basta galingan ninyo! Kung anong ginagawa ninyo, hindi ko pipiliting maging Tomista kayo [laughs]. Kung ano ang ginagawa ninyo ... At saka h'wag kayong matatakot sa wika. Kung magustuhan mo, halimbawa, si Kant, ang hamon sa iyo ay pag-aralan din ang wikang Aleman. $\mathrm{H}^{\prime}$ wag kang matatakot sa mga ganoon ding hamon. In fact, walang madali sa philosophy. $\mathrm{H}^{\prime}$ wag nating pipiliin ang isang pilosoper dahil lamang sa siya ay madali sa tingin natin. Pero, sa totoo lang, wala namang madaling pilosoper, ano?! Kung magustuhan mo ang isang pilosoper na ang wika ay French, o German,

(c) 2016 Emmanuel C. De Leon http://www.kritike.org/journal/issue 18/de leon june2016.pdf

ISSN 1908-7330 


\section{INTERVIEW WITH ROMUALDO ABULAD}

o Chinese, o kung anuman, kailagan mong hamunin ang sarili mo. Ang mahalaga ay mag-excel ka sa larangan na 'yan. Kailangang makita mo kung saan kang larangan ng pilosopiya makakapag-ambag. Everybody should challenge himself. Money is secondary.

De Leon: Maraming salamat po, Br. Romy. Malaking tulong po ito sa aming nagsisimula pa lamang.

Abulad: Sige. Goodluck sa iyo. Ph.D. na ba ang tinatapos mo?

De Leon: Opo.

Abulad: Ano ang talagang topic na gusto mong sulatin?

De Leon: Naiisip ko pong idokumento ang mga pamana ng mga pangunahing Tomasinong pilosoper sa takbo ng pamimilosopiya sa Pilipinas.

Abulad: Kasama pala ako diyan. Hindi ko alam 'yan. Salamat naman at isinama mo ako [laughs].

De Leon: Maraming maraming salamat po ulit.

End of Interview

Bibliography of Leading Thomasian Philosophers

\begin{tabular}{|c|c|c|}
\hline $\begin{array}{l}\text { Mga Pangunahing } \\
\text { Tomasinong } \\
\text { Pilosoper (1945- } \\
\text { Kasalukuyan) }\end{array}$ & Pamagat ng mga Akda & $\begin{array}{c}\text { Bilang ng } \\
\text { citations } \\
\text { base sa } \\
\text { Google } \\
\text { Scholar }\end{array}$ \\
\hline \multirow[t]{4}{*}{ 1. Romualdo Abulad } & $\begin{array}{l}\text { 1. "What is Hermeneutics?" in Kritike: An Online Journal of } \\
\text { Philosophy, 1:2 (December 2007), 11-23. }\end{array}$ & 19 \\
\hline & $\begin{array}{l}\text { 2. "Contemporary Filipino Philosophy," in Karunungan: The } \\
\text { Official Journal of the Philippine Academy of Philosophical Research } \\
\text { (1988), 1-13. }\end{array}$ & 12 \\
\hline & $\begin{array}{l}\text { 3. "Options for a Filipino Philosophy", in Karunungan, De La } \\
\text { Salle University (1984), 17-30. }\end{array}$ & 6 \\
\hline & $\begin{array}{l}\text { 4. "Kant's View of Science", in Sophia, De La Salle University, } \\
\text { vol. XVII, no. } 1 \text { (Aug 1987), 8-18. }\end{array}$ & 3 \\
\hline
\end{tabular}




\begin{tabular}{|c|c|c|}
\hline & $\begin{array}{l}\text { 5. "Origen's Contra Celsum: A Touch of Postmodernism", in } \\
\text { Diwa: Studies in Philosophy and Theology, Divine Word } \\
\text { Seminary, vol. XXII, no. } 1 \text { (May 1997), 26-40. }\end{array}$ & 2 \\
\hline & $\begin{array}{l}\text { 6. Ensayklopidiya ng Pilosopiya (co-authors: Quito, Timbreza, } \\
\text { Reyes), De La Salle University Press, } 1993 .\end{array}$ & 2 \\
\hline & $\begin{array}{l}\text { 7. "The Filipino as a Philosopher in Search of Originality", in } \\
\text { Karunungan, De La Salle University, vol. II, 1-25. }\end{array}$ & 1 \\
\hline & $\begin{array}{l}\text { 8. "The Mystical Philosophy of St. John of the Cross", in } \\
\text { Karunungan, De La Salle University, vol. 9, no. } 1 \text { (1992), 40-58. }\end{array}$ & 0 \\
\hline & $\begin{array}{l}\text { 9. "Challenge, Chance and Change: Dynamics of Lesson } \\
\text { Planning in the Philippine Secondary Schools" (co-author: H. } \\
\text { Guru) }\end{array}$ & 0 \\
\hline & $\begin{array}{l}\text { 10. "Post-Machiavelli", in Karunungan, University of Sto. } \\
\text { Tomas, vol. } 18 \text { (2001), 91-100. }\end{array}$ & 0 \\
\hline & $\begin{array}{l}\text { 11. "The Philosophical Bases of Values Education", in The } \\
\text { DLSU Graduate Journal, De La Salle University, vol. 12, no. } 2 \\
\text { (1987), 145-158. }\end{array}$ & 0 \\
\hline & $\begin{array}{l}\text { 12. "The Future of Ethics: A Postmodern View", in Diwa: } \\
\text { Studies in Philosophy and Theology, Divine Word Seminary - } \\
\text { Tagaytay, vol. XXVII, no. } 1 \text { (May 2002), 14-31. }\end{array}$ & 0 \\
\hline & $\begin{array}{l}\text { 13. "Edith Stein on Being Human as Grounded and Supported } \\
\text { by Eternal Being (co-author: J. Conrado Estafia) }\end{array}$ & 0 \\
\hline & $\begin{array}{l}\text { 14. Book Review: The Way of Greater Self Constructing a } \\
\text { Theology Around a Filipino Myth }\end{array}$ & 0 \\
\hline & $\begin{array}{l}\text { 15. "Science, Philosophy, Religion", in Diwa, Divine Word } \\
\text { Seminary - Tagaytay City, vol. XXIII, no. 2, pp. 99-125. }\end{array}$ & 0 \\
\hline & $\begin{array}{l}\text { 16. "Moving Forward, Moving Backward: Politics of } \\
\text { Curriculum Development in the Philippine Secondary } \\
\text { Schools" (co-author: H. Guru) }\end{array}$ & 0 \\
\hline & $\begin{array}{l}\text { 17. “Kalooban Mo, Kagustuhan Ko", in Malay, De La Salle } \\
\text { University, vol. VI, no. } 1 \text { (Oct 1986- May 1987), 40-47. }\end{array}$ & 0 \\
\hline & $\begin{array}{l}\text { 18. "Saan Ba Nanggaling ang Tao?", in Malay, De La Salle } \\
\text { University, vol. 2, no. } 1 \text { (1982). }\end{array}$ & 0 \\
\hline & $\begin{array}{l}\text { 19. "Ulat Tungkol sa Sanggunian at Leksikon ng Pilosopiya" } \\
\text { (co-authors: Quito, Timbreza, Reyes), in Malay, De La Salle } \\
\text { University, vol. 3, no. } 2 \text { (July 1983), 157-173. }\end{array}$ & 0 \\
\hline & $\begin{array}{l}\text { 20. "Ang Kasaysayan ng Pilosopiya and Ang Pilosopiya sa } \\
\text { Diwang Pilipino", by Emerita S. Quito," in Sophia, De La Salle } \\
\text { University, (Oct 1974-Jan 1975). [Book review]. }\end{array}$ & 0 \\
\hline & $\begin{array}{l}\text { 21. "Ang Pilosopiyang Pranses sa Pananaw ng Isang Pilipino", } \\
\text { in Karunungan, vol. } 8 \text { (1991), 65-89. }\end{array}$ & 0 \\
\hline & $\begin{array}{l}\text { 22. "Kant and Postmodernism", in Phavisminda Journal, } \\
\text { University of San Carlos, vol. } 2 \text { (May 1998), 32-51. }\end{array}$ & 0 \\
\hline & $\begin{array}{l}\text { 23. "Postmodern Critique of the Ethics of Postmodernism", in } \\
\text { Karunungan, University of Santo Tomas, vol. } 19 \text { (2002), 65-80. }\end{array}$ & 0 \\
\hline
\end{tabular}




\section{INTERVIEW WITH ROMUALDO ABULAD}

\begin{tabular}{|c|c|c|}
\hline & $\begin{array}{l}\text { 24. "God and Postmodernity", in Karunungan, University of } \\
\text { Santo Tomas, vol. } 19 \text { (2002), 5-13. }\end{array}$ & 0 \\
\hline & $\begin{array}{l}\text { 25. "What is Postmodernism?", in Karunungan, University of } \\
\text { Santo Tomas, vol. } 17 \text { (2000), 34-54. }\end{array}$ & 0 \\
\hline & Mga Akda na Hindi Nakalagay sa Google Scholar & \\
\hline & 1. "Bayani" (Karunungan, 1997) & \\
\hline & 2. "Freedom in Times of Crisis" (Unitas, 2001) & \\
\hline & 3. "A Philosopher's View of EDSA" (Karunungan, 2001) & \\
\hline & 4. “Anthrosophy of Rudolf Steiner" (Sophia, 1991) & \\
\hline & $\begin{array}{l}\text { 5. "Facing the Moral Challenge of the Postmodern Times" } \\
\text { (Karunungan, 1999) }\end{array}$ & \\
\hline & $\begin{array}{l}\text { 6. "Links Between East and West in the Philosophies of } \\
\text { Shankara and Kant" (Dissertation, 1978) }\end{array}$ & \\
\hline & $\begin{array}{l}\text { 7. “Ang Pilosopiya ng Salapi ni Georg Simmel” (Karunungan, } \\
\text { 1989) }\end{array}$ & \\
\hline & $\begin{array}{l}\text { 8. "The Significance of Kong Zi in the Age of Postmodernism" } \\
\text { (Karunungan, 2003) }\end{array}$ & \\
\hline & $\begin{array}{l}\text { 9. Two Filipino Thomasian Philosophers on Postmodernism (co- } \\
\text { editor: Alfredo Co) }\end{array}$ & \\
\hline & 10. “What is Philosophy?” (Karunungan, 1998) & \\
\hline & $\begin{array}{l}\text { 11. Criticism and Eternal Peace: Kant's Critique of Pure Reason as } \\
\text { the Method of Scientific Metaphysics, Alexander von Humboldt } \\
\text { Stiftung, University of Hamburg, } 1980 .\end{array}$ & \\
\hline & $\begin{array}{l}\text { 12. The Future of Man: The Philosophical Viewooint, De La Salle } \\
\text { University, } 1975 .\end{array}$ & \\
\hline & $\begin{array}{l}\text { 13. Introduction to Philosophy, vol. 1: Metaphysics, Theodicy, } \\
\text { Cosmology (with C. Ceniza), University of Santo Tomas, } 2001 .\end{array}$ & \\
\hline & $\begin{array}{l}\text { 14. Occasional Paper No. 4: Ang Pulitika ni Quezon at Iba Pang } \\
\text { Sanaysay, ni Marcelino A. Foronda (patnugot), De La Salle } \\
\text { University, } 1977 .\end{array}$ & \\
\hline & $\begin{array}{l}\text { 15. Readings in Filipino Philosophy, The Department of } \\
\text { Philosophy, De La Salle University, } 1990 .\end{array}$ & \\
\hline & $\begin{array}{l}\text { 16. The Status of Metaphysics in Kant's Critique of Pure Reason, } \\
\text { Ateneo de Manila University, } 1975 \text { (unpublished thesis). }\end{array}$ & \\
\hline & $\begin{array}{l}\text { 17. Toward a Reconstruction of Christology in the Context of } \\
\text { Postmodernity, Divine Word Seminary - Tagaytay City, } 2003 \\
\text { (unpublished thesis) }\end{array}$ & \\
\hline \multirow[t]{3}{*}{ 2. Alfredo Co } & $\begin{array}{l}\text { 1. "Doing Philosophy in the Philippines: Fifty Years Ago and } \\
\text { Fifty Years From Now", in Across the Philosophical Silk Road: A } \\
\text { Festschrift in Honor of Alfredo P. Co (Manila: University of } \\
\text { Santo Tomas Publishing House, 2004), 49-62. }\end{array}$ & 4 \\
\hline & $\begin{array}{l}\text { 2. "Elements of Chinese Thought in the Filipino Mind", in } \\
\text { Sophia, vol. } 5 \text { (1988), 27-34. }\end{array}$ & 4 \\
\hline & $\begin{array}{l}\text { 3. Philosophy of Ancient China (Manila: University of Santo } \\
\text { Tomas Publishing House, 2002). }\end{array}$ & 4 \\
\hline
\end{tabular}




\section{E. DE LEON 23}

\begin{tabular}{|c|c|c|}
\hline & $\begin{array}{l}\text { 4. The Blooming of a Hundred Flowers: Philosophy of Ancient } \\
\text { China (Manila: University of Santo Tomas Publishing House, } \\
1992 \text { and 2002). }\end{array}$ & 5 \\
\hline & $\begin{array}{l}\text { 5. "In the Beginning.... A Petit Personal Historical Narrative } \\
\text { of the Beginning of Philosophy in the Philippines", in Unitas: } \\
\text { A Quarterly Scholarly Journal for Arts and Sciences, vol. } 79 \\
\text { (2006). }\end{array}$ & 2 \\
\hline & $\begin{array}{l}\text { 6. "Filipino Philosophers in a Dialogue on Filipino Culture (A } \\
\text { Report)", in Karunungan, vol. } 9 \text { (2011). }\end{array}$ & 1 \\
\hline & $\begin{array}{l}\text { 7. “Chinese Thought and the Birth of Postmodern Culture: } \\
\text { The Western Man's Early Encounter with China”, (Thailand: } \\
\text { Assumption University, 2015). }\end{array}$ & 0 \\
\hline & $\begin{array}{l}\text { 8. “The Future of Man: The Classical Chinese Vision”, in } \\
\text { DLSU Dialogue: An Interdisciplinary Journal for Cultural Studies, } \\
\text { vol. 14, no. } 2 \text { (2013). }\end{array}$ & 0 \\
\hline & $\begin{array}{l}\text { 9. ACTA: Proceedings of the Quadricentennial International } \\
\text { Philosophy Congress (co-editor: Paolo Bolanos), [Manila, } \\
\text { University of Santo Tomas Publishing House, 2012]. }\end{array}$ & 0 \\
\hline & 10. Thomism and Asian Culturess (co-editor: Paolo Bolanos) & 0 \\
\hline & $\begin{array}{l}\text { 11. “The Work of Lao Zi as Found in the Ma-Wang-Tul } \\
\text { Archeological Excavations”, in Karunungan, vol. 8, no.1 } \\
\text { (Manila: University of Santo Tomas Publishing House, 2011). }\end{array}$ & 0 \\
\hline & $\begin{array}{l}\text { 12. Across the Philosophical Silk Road: A Festschrift in Honor of } \\
\text { Alfredo P. Co (Manila: University of Santo Tomas Publishing } \\
\text { House, 2009). }\end{array}$ & 0 \\
\hline & $\begin{array}{l}\text { 13. Across the Philosophical Silk Road: Under the Bo-tree, on the } \\
\text { Lotus Flower, Philosophy of the Compassionate Buddha (Manila: } \\
\text { University of Santo Tomas Publishing House, 2009). }\end{array}$ & 0 \\
\hline & $\begin{array}{l}\text { 14. Philosophy of the Compassionate Buddha: Under the Bo-tree--on } \\
\text { the Lotus Flower (Manila: University of Santo Tomas } \\
\text { Publishing House, 2003). }\end{array}$ & 0 \\
\hline & Mga Akda na Hindi Nakalagay sa Google Scholar & \\
\hline & $\begin{array}{l}\text { 1. Ang Tao at ang Pagkatao sa Pag-iisip Pilipino" } \\
\text { (Karunungan, 1998) }\end{array}$ & \\
\hline & $\begin{array}{l}\text { 2. “Classical Roots of Contemporary Chinese Politics" (Unitas, } \\
\text { 1985) }\end{array}$ & \\
\hline & 3. "The Dialectics of Tolerance" (Karunungan, 1997) & \\
\hline & 4. "Edengram as an Approach to Confucianism" & \\
\hline & $\begin{array}{l}\text { 5. "The Meeting of the East and West: A Story of the Class of } \\
\text { Cultures, Humiliation of a Civilization and the Restoration of } \\
\text { Pride (Karunungan, 2001) }\end{array}$ & \\
\hline & $\begin{array}{l}\text { 6. Across the Ancient Philosophical World: Essays in Comparative } \\
\text { Philosophy (UST Publishing House, 2015) }\end{array}$ & \\
\hline & $\begin{array}{l}\text { 7. "An Aesthetic Evaluation of Chinese Art" (UST Journal of } \\
\text { Graduate Research, 1987) }\end{array}$ & \\
\hline
\end{tabular}




\section{INTERVIEW WITH ROMUALDO ABULAD}

\begin{tabular}{|c|c|}
\hline & $\begin{array}{l}\text { 8. "An Man Created God: Understanding Postmodern Faith" } \\
\text { (Karunungan, 2002) }\end{array}$ \\
\hline & $\begin{array}{l}\text { 9. “The Book of Tao: A Work on Mysticism, a Political } \\
\text { Treatise, or a Military Treatise?" (Karunungan, 1986) }\end{array}$ \\
\hline & $\begin{array}{l}\text { 10. "Buddhism, Nihilism and the Contemporary Quest for a } \\
\text { Meaning in Life" (MA Thesis, 1974) }\end{array}$ \\
\hline & $\begin{array}{l}\text { 11. “Chinese Miniature Carvings" (UST Journal of Graduate } \\
\text { Research, 1989) }\end{array}$ \\
\hline & $\begin{array}{l}\text { 12. “Confucian Model for a Filipino Philosophy” } \\
\text { (Karunungan, 1990) }\end{array}$ \\
\hline & $\begin{array}{l}\text { 13. "Confucius: The Man, the Humanist at the Turn of a New } \\
\text { Millennium" (Karunungan, 1999) }\end{array}$ \\
\hline & $\begin{array}{l}\text { 14. "A Critical Reflection on Rene Descartes' 'Rules for the } \\
\text { Direction of the Mind"” (Unitas, 1997) }\end{array}$ \\
\hline & $\begin{array}{l}\text { 15. "Ethics and the Fears and Wonders of Human Cloning" } \\
\text { (Unitas, 1998) }\end{array}$ \\
\hline & $\begin{array}{l}\text { 16. "Expanding Worldview in a Shrinking Planet: Reading } \\
\text { Postmodernism in the Age of Globalization" (Karunungan, } \\
\text { 2002) }\end{array}$ \\
\hline & $\begin{array}{l}\text { 17. “Ang Hedonismo ni Yang Chu ng Antigong Tsina” } \\
\text { (Karunungan, 1989) }\end{array}$ \\
\hline & $\begin{array}{l}\text { 18. “Isang Komentaryo sa Paglulunsad ng Aklat na } \\
\text { Pinamagatang Commentaries on Moral Philosophy" } \\
\text { (Karunungan, 1999) }\end{array}$ \\
\hline & $\begin{array}{l}\text { 19. “Man's Search for Fulfillment: An Oriental Perspective" } \\
\text { (PhD. Dissertation, 1976) }\end{array}$ \\
\hline & $\begin{array}{l}\text { 20. "Media and Communication Technology and the Pitfalls } \\
\text { of Modern Populism: The Case of the Impeachment Trial of } \\
\text { Ex-President Joseph Ejercito Estrada of the Republic of the } \\
\text { Philippines" (Karunungan, 2001) }\end{array}$ \\
\hline & $\begin{array}{l}\text { 21. "Oracle Bones: Ancient China's Tool for Divination" (UST } \\
\text { Journal of Graduate Research, 1985) }\end{array}$ \\
\hline & $\begin{array}{l}\text { 22. "The Problem of Truth in Classical Chinese Philosophy" } \\
\text { (UST Journal of Graduate Research, 1988) }\end{array}$ \\
\hline & $\begin{array}{l}\text { 23. "A Reflection on the Hindu Concept of Niskamakarma" } \\
\text { (Karunungan, 1999) }\end{array}$ \\
\hline & $\begin{array}{l}\text { 24. "Some Reflections of the Philosophy of Man in Ancient } \\
\text { Confucianism" (UST Journal of Graduate Research, 1989) }\end{array}$ \\
\hline & $\begin{array}{l}\text { 25. "Some Reflections on the Thanatology of Zhuang Zi" } \\
\text { (Karunungan, 2000) }\end{array}$ \\
\hline & $\begin{array}{l}\text { 26. "Sun Zi's Art of War and His Message of Peace" (Unitas, } \\
\text { 1988) }\end{array}$ \\
\hline & $\begin{array}{l}\text { 27. “Why Death Penalty? ... Why not Death Penalty?" } \\
\text { (Karunungan, 2000) }\end{array}$ \\
\hline
\end{tabular}




\section{E. DE LEON 25}

\begin{tabular}{|c|c|c|}
\hline 3. Florentino Hornedo & $\begin{array}{l}\text { 1. Taming the Wind: Ethno-cultural history on the Ivatan of the } \\
\text { Batanes Isles (Manila: University of Santo Tomas Publishing } \\
\text { House, 2000). }\end{array}$ & 11 \\
\hline & $\begin{array}{l}\text { 2. Culture and Community in the Philippine Fiesta and Other } \\
\text { Celebrations (Manila: University of Santo Tomas Publishing } \\
\text { House, 2000). }\end{array}$ & 6 \\
\hline & $\begin{array}{l}\text { 3. "Ivatan Oral Traditions: A Survey", in Philippine Studies: } \\
\text { Historical and Ethnographic Viewpoints, vol. 25, no. } 4 \text { (Quezon } \\
\text { City: Ateneo de Manila University Press, 1977), 384-419. }\end{array}$ & 6 \\
\hline & $\begin{array}{l}\text { 4. Laji: An Ivatan Folk Lyric Tradition (Manila: University of } \\
\text { Santo Tomas Publishing House, 1979). }\end{array}$ & 6 \\
\hline & $\begin{array}{l}\text { 5. "The World and the Ways of the Ivatan Anitu", in Philippine } \\
\text { Studies, vol. 28, no. } 1 \text { (Quezon City: Ateneo de Manila } \\
\text { University Press, 1980), 21-58. }\end{array}$ & 6 \\
\hline & $\begin{array}{l}\text { 6. “'Induyan': An Amburayan Migration Song”, in Philippine } \\
\text { Studies (Quezon City: Ateneo de Manila University Press, } \\
\text { 1990), 358-368. }\end{array}$ & 4 \\
\hline & $\begin{array}{l}\text { 7. Christian Education: Becoming Person-for-others, Essays in } \\
\text { Philosophy of Education (Manila: University of Santo Tomas } \\
\text { Publishing House, 1995). }\end{array}$ & 4 \\
\hline & 8. "Development Begins with Self-help", in Payuhwan (1989). & 4 \\
\hline & $\begin{array}{l}\text { 9. "The Alien in the Bakun-Amburayan River Valley Oral } \\
\text { Literature", in Philippine Studies (Quezon City: Ateneo de } \\
\text { Manila University Press, 1990), 199-225. }\end{array}$ & 3 \\
\hline & $\begin{array}{l}\text { 10. "Notes on Batanes and the Ivatan Way of Life", (1971), 5- } \\
\text { 46. }\end{array}$ & 3 \\
\hline & $\begin{array}{l}\text { 11. Batanes Ethnographic History - A Survey (Quezon City: } \\
\text { Ateneo de Manila University Press, 1976). }\end{array}$ & 3 \\
\hline & $\begin{array}{l}\text { 12. The Power to be: A Phenomenology of Freedom (Manila: } \\
\text { University of Santo Tomas Publishing House, 2000). }\end{array}$ & 3 \\
\hline & $\begin{array}{l}\text { 13. "The Persona in Philippine Folk Literature" in Reading } \\
\text { Popular Culture (Quezon City: Office of Research and } \\
\text { Publications, Ateneo de Manila University, 1991), 67-80. }\end{array}$ & 3 \\
\hline & $\begin{array}{l}\text { 14. "Truth, Man, and Martin Heidegger" in St. Louis } \\
\text { University Research Journal, vol. } 2 \text { (Baguio City, 1971), 1-18. }\end{array}$ & 3 \\
\hline & $\begin{array}{l}\text { 15. Ideas and Ideals: Essays in Filipino Cognitive History (Manila: } \\
\text { University of Santo Tomas Publishing House, } 2001 .\end{array}$ & 2 \\
\hline & $\begin{array}{l}\text { 16. The Favor of the Gods: Essays in Filipino Religions Thought and } \\
\text { Behavior (Manila: University of Santo Tomas Publishing } \\
\text { House, 2001). }\end{array}$ & 2 \\
\hline & $\begin{array}{l}\text { 17. "Report in the Regional Seminar for Culture Personnel in } \\
\text { Asia and the Pacific" }\end{array}$ & 2 \\
\hline & $\begin{array}{l}\text { 18. "Death and After Death: Ivatan Beliefs and Practices", in } \\
\text { Philippine Studies, vol. } 42 \text {, no. } 4 \text { (Quezon City: Ateneo de } \\
\text { Manila University Press, 1994), 509-527. }\end{array}$ & 2 \\
\hline
\end{tabular}

(c) 2016 Emmanuel C. De Leon

http://www.kritike.org/journal/issue 18/de leon june2016.pdf

ISSN 1908-7330 


\section{INTERVIEW WITH ROMUALDO ABULAD}

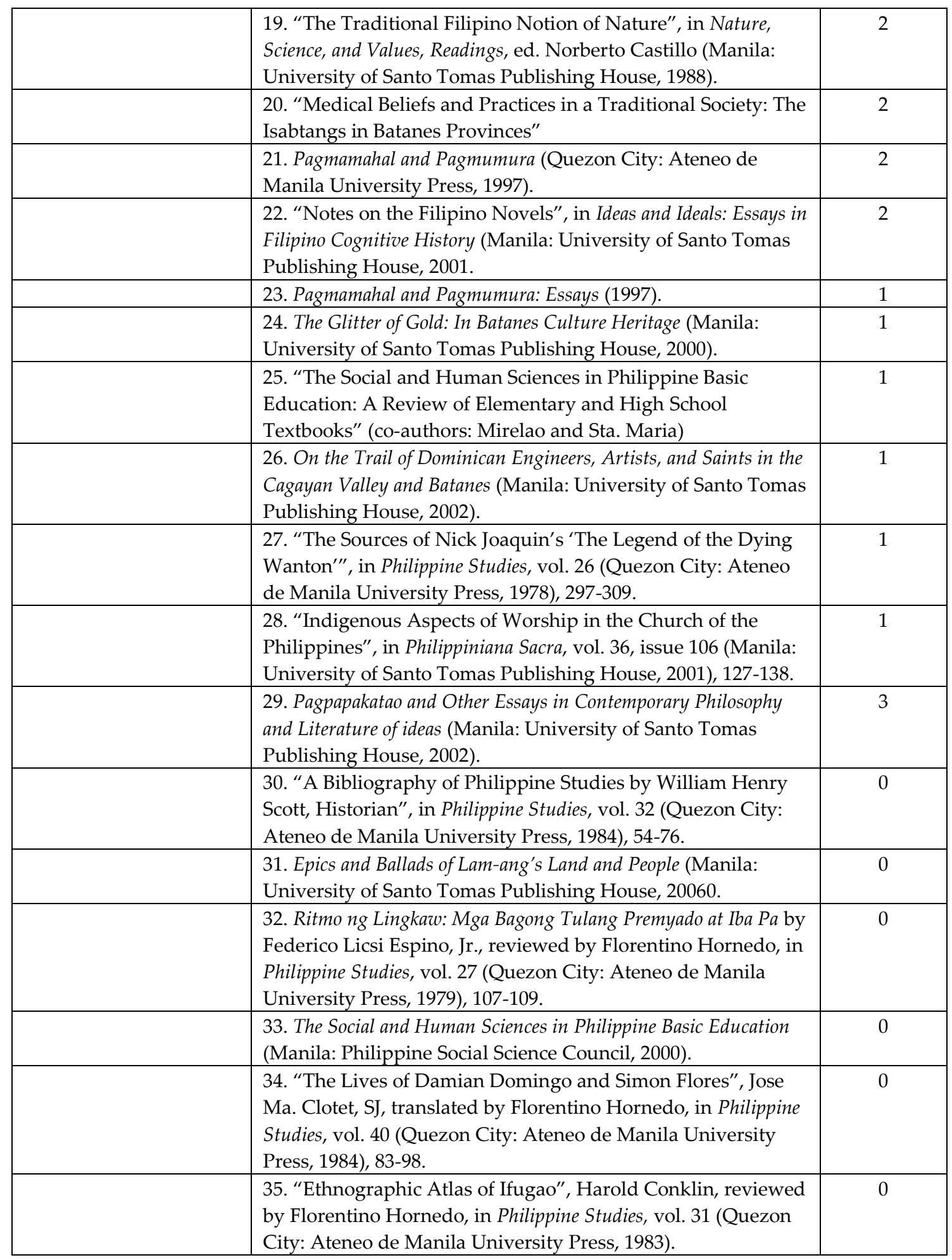

(C) 2016 Emmanuel C. De Leon

http://www.kritike.org/journal/issue 18/de leon june2016.pdf

ISSN 1908-7330

(cc) EY-NC-ND 


\section{E. DE LEON 27}

\begin{tabular}{|c|c|c|}
\hline & $\begin{array}{l}\text { 36. "Towards an Hermeneutics of Pagmamahal and } \\
\text { Pagmumura", in Saint Louis University Research Journal, vol. } 16 \\
\text { (June 1985), 72-89. }\end{array}$ & 0 \\
\hline & $\begin{array}{l}\text { 37. "Batanes at the Start of the American Era according to Jose } \\
\text { Serres", in Ad Veritatem, vol. 4, no. } 2 \text { (March 2005), 459-470. }\end{array}$ & 0 \\
\hline & $\begin{array}{l}\text { 38. “Journey to Majayjay", Paul de la Gironierre, reviewed by } \\
\text { Florentino Hornedo, in Philippine Studies, vols. 32-33 (Quezon } \\
\text { City: Ateneo de Manila University Press, 1984), 239-241. }\end{array}$ & 0 \\
\hline & $\begin{array}{l}\text { 39. "Epiphany and Celebration in Asian Life and Aesthetics", } \\
\text { in Luz y Saber, vol. 1, no. } 1 \text { (2007). }\end{array}$ & 0 \\
\hline & $\begin{array}{l}\text { 40. "Bago Mythology and the Ecosystem" in Unitas (Manila: } \\
\text { University of Santo Tomas Publishing House, 1993). }\end{array}$ & 0 \\
\hline & 41. "The Bago Ballad of Delnagen and Anmusan Lumawig" & 0 \\
\hline & $\begin{array}{l}\text { 42. Back to the Things Themselves: Selected Essays in Recent } \\
\text { Western Philosophy (Editor, 2003) }\end{array}$ & 0 \\
\hline & $\begin{array}{l}\text { 43. Book Review: Lorenzo Ruiz, the Protomartyr of the } \\
\text { Philippines, and His Companions (by Villaroel) }\end{array}$ & 0 \\
\hline & 44. “Battle of La Naval: Rage of Waves Fury of Faith" & 0 \\
\hline & Mga Akda na Hindi Nakalagay sa Google Scholar & \\
\hline & $\begin{array}{l}\text { 1. “The Agamaniyog Folktales: Mindanao Art and Culture" } \\
\text { (Unitas, 1980) }\end{array}$ & \\
\hline & 2. “Alejandro G. Abadilla: 'Ako ang Daigdig' (Unitas, 2007) & \\
\hline & $\begin{array}{l}\text { 3. "Ang Humanismo sa Panahon ng Reasinyento" (Unitas, } \\
\text { 2003) }\end{array}$ & \\
\hline & 4. "Ang Klasisismo at ang Panitikang Pilipino" (Unitas, 2003) & \\
\hline & $\begin{array}{l}\text { 5. “Ang Literatura sa Silid-aralan: Ilang Kwadradong } \\
\text { Konseptwal Para sa Pagtuturo ng Literatura" (Unitas, 1999) }\end{array}$ & \\
\hline & $\begin{array}{l}\text { 6. "Bibliographic Study of Fray Tomas Ortiz Arte y Reglas de } \\
\text { la Lengua" (Colloquia Manilana, 1993) }\end{array}$ & \\
\hline & $\begin{array}{l}\text { 7. Book Reviews: Myths and Symbols Philippines (Unitas, } \\
\text { 1980) }\end{array}$ & \\
\hline & $\begin{array}{l}\text { 8. "Challenges to Literacy Education in the } 21^{\text {st }} \text { Century" } \\
\text { (Unitas, 1999) }\end{array}$ & \\
\hline & $\begin{array}{l}\text { 9. "The Changing Core Themes of Filipino Nationalism and } \\
\text { their Literacy" (Unitas, 1989) }\end{array}$ & \\
\hline & $\begin{array}{l}\text { 10. "The Classical and the Romantic World Views: Their } \\
\text { Reflection in the Philosophy of Art" (UST Journal of Graduate } \\
\text { Research, 1984) }\end{array}$ & \\
\hline & 11. “The Condition of the Present" (Unitas, 1999) & \\
\hline & 12. “Crisis of Law” (Unitas, 1985) & \\
\hline & 13. "'Cultural Studies' and Hegemony" (Karunungan, 2002) & \\
\hline & 14. “Cultural-Rooted Education” (Unitas, 2004) & \\
\hline & $\begin{array}{l}\text { 15. “Current Trends in Literary Theory and Criticism" } \\
\text { (Unitas, 1997) }\end{array}$ & \\
\hline & $\begin{array}{l}\text { 16. "The Development of Jean-Paul Sartre's Concept of } \\
\text { Human Freedom" (The Thomasian Philosopher, 2010) }\end{array}$ & \\
\hline
\end{tabular}




\begin{tabular}{|c|c|c|}
\hline & 17. "Ethnographic Atlas of Ifugao" (Unitas, 1982) & \\
\hline & $\begin{array}{l}\text { 18. “Excellent Book to Clarify All Clarifications" (Unitas, } \\
\text { 1996) }\end{array}$ & \\
\hline & 19. “The Favor of the Gods” (Unitas, 1997) & \\
\hline & 20. “Filipino Values (Institutional vs. Personal) [Unitas, 1999) & \\
\hline & $\begin{array}{l}\text { 21. “Fr. Bernardino Melenderas 'Antologia Poenca' and 19th } \\
\text { Century Philippine Literary Relations” (Unitas, 1990) }\end{array}$ & \\
\hline & $\begin{array}{l}\text { 22. "French Revolution and the Rise of the Filipino } \\
\text { Nationalism" (Unitas, 1993) }\end{array}$ & \\
\hline & 23. “George Seferis” (Unitas, 1999) & \\
\hline & $\begin{array}{l}\text { 24. "Honest to Rizal and to the Filipino People" (Philippiniana } \\
\text { Sacra, 1984) }\end{array}$ & \\
\hline & 25. “Hotline to Heaven” (Life Today, 1992) & \\
\hline & $\begin{array}{l}\text { 26. "Indigenous Aspects of Worship in the Church of the } \\
\text { Philippines" (Unitas, 1997) }\end{array}$ & \\
\hline & $\begin{array}{l}\text { 27. “Jose Calatrava Remebers the Guerila Days in Batanes in } \\
\text { 1945" }\end{array}$ & \\
\hline & 28. “Kabbuni” (Unitas, 1993) & \\
\hline & 29. “Kulturang Popular” (Unitas, 1993) & \\
\hline & $\begin{array}{l}\text { 30. "Leadership/People Mutal Alienation as Cause of Peace" } \\
\text { (Unitas, 1983) }\end{array}$ & \\
\hline & 31. “Literary Theory and Literary Pedagogy” (Unitas, 2002) & \\
\hline & 32. "Methods of Teaching and Learning" (Unitas, 2001) & \\
\hline & $\begin{array}{l}\text { 33. “Notes on Postmodern Literary Theory and Criticism" } \\
\text { (Unitas, 1995) }\end{array}$ & \\
\hline & 34. “Pagpapakatao” (UST Journal of Graduate Research, 1990) & \\
\hline & $\begin{array}{l}\text { 35. “Philippine Literary Aesthetics and Social Class" (Unitas, } \\
\text { 2005) }\end{array}$ & \\
\hline & $\begin{array}{l}\text { 36. “Professionalism and the UST Academician” (Unitas, } \\
\text { 1991) }\end{array}$ & \\
\hline & $\begin{array}{l}\text { 37. “Reforestation in Traditional Ivatan Yam Cultivation" } \\
\text { (Unitas, 1995) }\end{array}$ & \\
\hline & 38. “Religious Art in Batanes” (Philippiniana Sacra, 1983) & \\
\hline & $\begin{array}{l}\text { 39. "The Right to Education and Culture in the Basic } \\
\text { Document of the United Nations" (Unitas, 1990) }\end{array}$ & \\
\hline & $\begin{array}{l}\text { 40. "The School and the Family: Towards a Culture of Peace" } \\
\text { (Unitas, 2003) }\end{array}$ & \\
\hline & 41. "Social Sciences" (Unitas, 1989) & \\
\hline & 42. "Some Notes on the Philippine Flag" (Unitas, 1996) & \\
\hline & 43. “Stone in Batanes Cultural History” (Unitas, 2000) & \\
\hline & $\begin{array}{l}\text { 44. "Survey of the History of Urbanization and Religion- } \\
\text { Related Arts in the Cagayan Valley-Batanes Region from the } \\
16^{\text {th }} \text { Century" (Unitas, 1994) }\end{array}$ & \\
\hline & $\begin{array}{l}\text { 45. "The Theme of Loneliness and Hollowness in Modern } \\
\text { Western Literature" (Unitas, 1999) }\end{array}$ & \\
\hline
\end{tabular}




\begin{tabular}{|c|c|c|}
\hline & $\begin{array}{l}\text { 46. “Three Essays on Values and Education” (UST Journal of } \\
\text { Graduate Research, 1989) }\end{array}$ & \\
\hline & $\begin{array}{l}\text { 47. “Today's Native was Yesterday's Visitor: Filipinicity in } \\
\text { Folk and Popular Literature” (UST Journal of Graduate } \\
\text { Research, 1988) }\end{array}$ & \\
\hline & $\begin{array}{l}\text { 48. “Wittgenstein's Language-games" (The Thomasian } \\
\text { Philosopher, 2010) }\end{array}$ & \\
\hline & $\begin{array}{l}\text { 49. “World, the Sacred, and Traditional Filipino Societies” } \\
\text { (Unitas, 1991) }\end{array}$ & \\
\hline & $\begin{array}{l}\text { 50. "Youth and the Challenge of a Fast Expanding Global } \\
\text { Horizon" (Unitas, 1996). }\end{array}$ & \\
\hline \multirow[t]{16}{*}{ 4. Leonardo Mercado } & $\begin{array}{l}\text { 1. Elements of Filipino Philosophy (Tacloban City: Divine Word } \\
\text { University Publication, 1974). }\end{array}$ & 68 \\
\hline & $\begin{array}{l}\text { 2. Elements of Filipino Theology (Tacloban City: Divine Word } \\
\text { University Publication, 1975). }\end{array}$ & 30 \\
\hline & 3. The Filipino Mind, published by CRVP (1994). & 27 \\
\hline & $\begin{array}{l}\text { 4. The Filipino Religious Psychology, editor (Tacloban City: } \\
\text { Divine Word University Publications, 1977). }\end{array}$ & 15 \\
\hline & $\begin{array}{l}\text { 5. Christ in the Philippines (Tacloban City: Divine Word } \\
\text { University Publications, 1981). }\end{array}$ & 13 \\
\hline & $\begin{array}{l}\text { 6. Applied Filipino Philosophy (Tacloban City: Divine Word } \\
\text { University Publications, 1977). }\end{array}$ & 12 \\
\hline & $\begin{array}{l}\text { 7. Elements of Filipino Ethics (Tacloban City: Divine Word } \\
\text { University Publications, 1979). }\end{array}$ & 10 \\
\hline & $\begin{array}{l}\text { 8. "Notes on the Filipino Philosophy of Work and Leisure”, in } \\
\text { Philippine Studies, vol. 22, nos. 1-2 (Quezon City: Ateneo de } \\
\text { Manila University Press, 1974), 71-80. }\end{array}$ & 7 \\
\hline & $\begin{array}{l}\text { 9. Inculturation and Filipino Theology (Manila: Divine Word } \\
\text { Publications, 1992). }\end{array}$ & 6 \\
\hline & $\begin{array}{l}\text { 10. Filipino Popular Devotions: The Interior Dialogue Between } \\
\text { Traditional Religions and Christianity, editor (Manila: Logos } \\
\text { Publications, Inc., 2000). }\end{array}$ & 4 \\
\hline & $\begin{array}{l}\text { 11. Filipino Thought on Man and Society, editor (Tacloban City: } \\
\text { Divine Word Publications, 1980). }\end{array}$ & 4 \\
\hline & 12. El Shaddai: A Study (Manila: Logos Publications, 2001). & 4 \\
\hline & $\begin{array}{l}\text { 13. "Filipino Thought”, in Philippine Studies, vol. 20, no. } 2 \\
\text { (Quezon City: Ateneo de Manila University Press, 1972), 207- } \\
272 .\end{array}$ & 5 \\
\hline & $\begin{array}{l}\text { 14. Mission and Dialogue: Theory and Practice, co-edited by } \\
\text { James Knight (Manila: Divine Word Publications, 1987). }\end{array}$ & 4 \\
\hline & $\begin{array}{l}\text { 15. "Loob, Body, Self, Bait", in The Filipino Mind (Washington } \\
\text { DC: The Council for Research in Values and Philosophy and } \\
\text { Divine Word Publications, 1994). }\end{array}$ & 4 \\
\hline & $\begin{array}{l}\text { 16. Essays on Filipino Philosophy (Manila: Logos Publications, } \\
\text { 2005). }\end{array}$ & 3 \\
\hline
\end{tabular}




\begin{tabular}{|c|c|c|}
\hline & $\begin{array}{l}\text { 17. Doing Filipino Theology, editor (Manila: Logos Publications, } \\
\text { Inc., 1995). }\end{array}$ & 2 \\
\hline & $\begin{array}{l}\text { 18. “The Filipino Image of God”, in Philippiniana Sacra, vol. 26, } \\
\text { issue78 (Manila: University of Santo Tomas Publishing } \\
\text { House, 1991), 401-415. }\end{array}$ & 3 \\
\hline & $\begin{array}{l}\text { 19. Working with Indigenous People: A Philippine Source Book } \\
\text { (Manila: Divine Word Publications, 1994). }\end{array}$ & \\
\hline & $\begin{array}{l}\text { 20. Philippine Muslim-Christian Dialogue, co-edited by Maxwell } \\
\text { Felicilda (Manila: Logos Publications, 1993). }\end{array}$ & 2 \\
\hline & $\begin{array}{l}\text { 21. Research Methods in Philippine Context, editor (Tacloban } \\
\text { City: Divine Word University Publications, 1983). }\end{array}$ & 2 \\
\hline & $\begin{array}{l}\text { 22. "Soul and Spirit in Filipino Thought", in Philippine Studies, } \\
\text { vol. 39, no. } 3 \text { (Quezon City: Ateneo de Manila University } \\
\text { Press, 1991), 287-302. }\end{array}$ & 2 \\
\hline & $\begin{array}{l}\text { 23. Legal Philosophy: Western, Eastern, E Filipino (Tacloban City: } \\
\text { Divine Word University Publications, 1984). }\end{array}$ & 2 \\
\hline & $\begin{array}{l}\text { 24. Filipino Psychology for Village Development, editor (Tacloban } \\
\text { City: Divine Word University Publications, 1980). }\end{array}$ & 2 \\
\hline & $\begin{array}{l}\text { 25. "Christian and Interfaith Spirituality", in Interpretations for } \\
\text { Peace in the Religions of the Near East (2007), } 7 .\end{array}$ & 1 \\
\hline & $\begin{array}{l}\text { 26. Towards an Asian Theology of Mission (Manila: Logos } \\
\text { Publications, 1995). }\end{array}$ & 1 \\
\hline & $\begin{array}{l}\text { 27. “Inculturation and Biblical Apostolate”, in Philippiniana } \\
\text { Sacra, vol. 26, issue } 76 \text { (Manila: University of Santo Tomas } \\
\text { Publishing House, 1991), 37-49. }\end{array}$ & 1 \\
\hline & $\begin{array}{l}\text { 28. “Reflections on Buut-Loob-Nakem”, in Philippine Studies, } \\
\text { vol. 20, no. } 4 \text { (Quezon City: Ateneo de Manila University } \\
\text { Press, 1972), 577-601. }\end{array}$ & 1 \\
\hline & Mga Akda na Hindi Nakalagay sa Google Scholar & \\
\hline & 1. "Bayani: The Filipino Concept of Hero" (Karunungan, 1997) & \\
\hline & 2. "El Shaddai and Inculturation" (Philippiniana Sacra, 2000) & \\
\hline & $\begin{array}{l}\text { 3. "The Erap Impeachment Trial: People Power } 2 \text { and Filipino } \\
\text { Legal Philosophy" (Karunungan, 2001) }\end{array}$ & \\
\hline & $\begin{array}{l}\text { 4. "The New Paradigm and Filipino Philosophy" } \\
\text { (Karunungan, 1998) }\end{array}$ & \\
\hline & 5. "A Notion of Philosophy” (Karunungan, 1998) & \\
\hline & 6. "Behind the Media Show" (World Mission, 2006) & \\
\hline & 7. “A Five-Century Devotion” (World Mission, 2008) & \\
\hline & 8. “A Poetic Salvation History” (World Mission, 2008) & \\
\hline & 9. “The Saint of Last Resort” (World Mission, 2007) & \\
\hline & 10. “Cross-cultural Ministry” (Philippiniana Sacra, 2008) & \\
\hline & $\begin{array}{l}\text { 11. "Dancing with the Music: Missionary Spirituality as } \\
\text { Experienced with the Indigenous Peoples and Community" } \\
\text { (Institute of Spirituality in Asia, 2008) }\end{array}$ & \\
\hline & 12. “Descartes and Ecology" (Unitas, 1996) & \\
\hline & 13. Dialogue and Faith: A Philippine View (Logos, 2009) & \\
\hline
\end{tabular}




\section{E. DE LEON 31}

\begin{tabular}{|c|c|c|}
\hline & 14. Explorations in Filipino Philosophy (Logos, 2009) & \\
\hline & 15. “Filipino Face of Christ” (Philippiniana Sacra, 1992) & \\
\hline & $\begin{array}{l}\text { 16. The Filipino Mind (Washington, D.C.: Council for Research } \\
\text { in Values and Philosophy, 1994) }\end{array}$ & \\
\hline & $\begin{array}{l}\text { 17. Filipino Religious Experience and Non-Biblical Revelation } \\
\text { (Logos, 1992) }\end{array}$ & \\
\hline & $\begin{array}{l}\text { 18. Filipino Religious Psychology (Divine Word University, } \\
\text { 1977) }\end{array}$ & \\
\hline & 19. "Holy in the Analects of Confucius" (Sophia, 1989) & \\
\hline & 20. “The Image of Filipino Faith” (World Mission, 2008) & \\
\hline & $\begin{array}{l}\text { 21. Inter-religious Explorations: The Challenge and Rewards of } \\
\text { Inter-Religious Dialogue (Logos, 2004) }\end{array}$ & \\
\hline & $\begin{array}{l}\text { 22. Inter-religious Stories and Experiences (Compiled and Edited, } \\
\text { Logos, 2000) }\end{array}$ & \\
\hline & $\begin{array}{l}\text { 23. “Kagandahan: Filipino Thought on Beauty, Truth, and } \\
\text { Good" (Karunungan, 1992) }\end{array}$ & \\
\hline & $\begin{array}{l}\text { 24. "Muslim and Christian Fundamentalism in the } \\
\text { Philippines" (Philippiniana Sacra, 1998) }\end{array}$ & \\
\hline & $\begin{array}{l}\text { 25. "The Role of the Religious in the Indigenization of Liturgy } \\
\text { and Prayer" (Boletin Eclesiastico de Filipinas, 1978) }\end{array}$ & \\
\hline & 26. "A Notion of Philosophy" (Karunungan, 1998) & \\
\hline & $\begin{array}{l}\text { 27. Old Cultures Renewed Religions: The Search for Cultural } \\
\text { Identity in a Changing World" (Logos, 2001) }\end{array}$ & \\
\hline & $\begin{array}{l}\text { 28. “On Evaluating Religious Phenomena" (Karunungan, } \\
\text { 2002) }\end{array}$ & \\
\hline & 29. “On Filipino Ethics” (Karunungan, 1999) & \\
\hline & $\begin{array}{l}\text { 30. "Paradigm Shifts and Religious Tolerance" (Philippiniana } \\
\text { Sacra, 1996) }\end{array}$ & \\
\hline & $\begin{array}{l}\text { 31. “Philippine Shamanism and Inculturation” (Philippiniana } \\
\text { Sacra, 1995) }\end{array}$ & \\
\hline & $\begin{array}{l}\text { 32. "Signs and Wonders from the Philippine Perspective" } \\
\text { (Karunungan, 2000) }\end{array}$ & \\
\hline & $\begin{array}{l}\text { 33. Towards and Asian Theology of Mission (Divine Word } \\
\text { Publications, 1995) }\end{array}$ & \\
\hline & $\begin{array}{l}\text { 34. Working with Indigenous People: A Philippine Sourcebook } \\
\text { (Divine Word, 1994) }\end{array}$ & \\
\hline \multirow[t]{4}{*}{ 5. Emerita Quito } & $\begin{array}{l}\text { 1. The State of Philosophy in the Philippines (Manila: De La Salle } \\
\text { University Press, 1983). }\end{array}$ & 12 \\
\hline & $\begin{array}{l}\text { 2. “The Ambivalence of Filipino Traits and Values” in } \\
\text { Karunungan (Sophia), (Manila: De La Salle University Press, } \\
\text { 1988). }\end{array}$ & 11 \\
\hline & $\begin{array}{l}\text { 3. The Philosophers of Hermeneutics (Manila: De La Salle } \\
\text { University Press, 1990). }\end{array}$ & 10 \\
\hline & $\begin{array}{l}\text { 4. The Merging Philosophy of East and West (Manila: De La Salle } \\
\text { University Press, 1991). }\end{array}$ & 5 \\
\hline
\end{tabular}




\section{INTERVIEW WITH ROMUALDO ABULAD}

\begin{tabular}{|c|c|c|}
\hline & $\begin{array}{l}\text { 5. “Volksgeist in Vernacular Literature", in A Life of } \\
\text { Philosophy: Fetschrift in Honor of Emerita Quito (Manila: De La } \\
\text { Salle University Press, 1990). }\end{array}$ & 6 \\
\hline & $\begin{array}{l}\text { 6. "Structuralism and the Filipino Volksgeist", in Dialogue, } \\
\text { vol. 18, no. } 2 \text { (Manila: De La Salle University Press, 1983). }\end{array}$ & 3 \\
\hline & $\begin{array}{l}\text { 7. Philosophy of Education for Filipinos, in A Life of Philosophy: } \\
\text { Festschrift in Honor of Emerita S. Quito (Manila: De La Salle } \\
\text { University, 1990). }\end{array}$ & 2 \\
\hline & $\begin{array}{l}\text { 8. Phenomenology: Edmund Husserl and Edith Stein (Manila: De } \\
\text { La Salle University Press, 2001). }\end{array}$ & 2 \\
\hline & $\begin{array}{l}\text { 9. Oriental Roots of Occidental Philosophy (Manila: De La Salle } \\
\text { University Press, 1975). }\end{array}$ & 2 \\
\hline & $\begin{array}{l}\text { 10. Ensayklopidiya ng Pilosopiya (co-authors: Abulad, Timbreza, } \\
\text { Reyes), De La Salle University Press, } 1993 .\end{array}$ & 2 \\
\hline & 11. A Life of Philosophy: Selected Works (1965-1988) & 1 \\
\hline & $\begin{array}{l}\text { 12. Three Women Philosophers (Manila: De La Salle University } \\
\text { Press, 1986). }\end{array}$ & 1 \\
\hline & $\begin{array}{l}\text { 13. Fundamentals of Ethics (Manila: De La Salle University } \\
\text { Press, 1989). }\end{array}$ & 1 \\
\hline & $\begin{array}{l}\text { 14. “The Role of the University in Changing Women's } \\
\text { Consciousness" in DLSU Dialogue: An Interdisciplinary Journal } \\
\text { for Cultural Studies, vol. 14, no, } 1 \text { (Manila: De La Salle } \\
\text { University Press, 1978). }\end{array}$ & 1 \\
\hline & $\begin{array}{l}\text { 15. St. John Baptist de la Salle and Education Today (Manila: De } \\
\text { La Salle University Press, 1992). }\end{array}$ & 1 \\
\hline & $\begin{array}{l}\text { 16. Critique of Historical Today (Manila: De La Salle University } \\
\text { Press, 2002). }\end{array}$ & 0 \\
\hline & $\begin{array}{l}\text { 17. "The Paradox of Freedom in Louis Lavelle" in } \\
\text { Unitas (Manila: University of Santo Tomas Publishing House, } \\
\text { 2003). }\end{array}$ & 0 \\
\hline & $\begin{array}{l}\text { 18. Herbert Marcuse and Contemporary Society (Manila: } \\
\text { University of Santo Tomas Publishing House, 1970). }\end{array}$ & 0 \\
\hline & $\begin{array}{l}\text { 19. Lectures on Comparative Philosophy (Manila: De La Salle } \\
\text { University Press, 1979). }\end{array}$ & 0 \\
\hline & $\begin{array}{l}\text { 20. "Address on a New Concept of Philosophy at the Opening } \\
\text { Exercises of Academic Year 1967-1968" }\end{array}$ & 0 \\
\hline & $\begin{array}{l}\text { 21. A Structural Approach to Philippine Epics (Manila: Museo ng } \\
\text { Kalinangan, 1989). }\end{array}$ & 0 \\
\hline & $\begin{array}{l}\text { 22. "The Best of a Decade of Sophia 1971-1981" (Manila: De La } \\
\text { Salle University Press). }\end{array}$ & 0 \\
\hline & $\begin{array}{l}\text { 23. "Robert Ardrey: Scientist or Philosopher?" in Unitas } \\
\text { (Manila: University of Santo Tomas Publishing House, 1971). }\end{array}$ & 0 \\
\hline & $\begin{array}{l}\text { 24. "Yoga and Christian Spirituality", in Unitas (Manila: } \\
\text { University of Santo Tomas Publishing House, 1978). }\end{array}$ & 0 \\
\hline & $\begin{array}{l}\text { 25. Four Essays in the Philosophy of History (Manila: De La Salle } \\
\text { University Press, 1979). }\end{array}$ & 0 \\
\hline
\end{tabular}




\section{E. DE LEON 33}

\begin{tabular}{|c|c|c|}
\hline & $\begin{array}{l}\text { 26. "Ulat Tungkol sa Sanggunian at Leksikon ng Pilosopiya" } \\
\text { (co-authors: Abulad, Timbreza, Reyes), in Malay, De La Salle } \\
\text { University, vol. 3, no. } 2 \text { (July 1983), 157-173. }\end{array}$ & 0 \\
\hline & $\begin{array}{l}\text { 27. "Philosophy of History: An Introduction." In DLSU } \\
\text { Dialogue: An Interdisciplinary Journal for Cultural Studies, vol. } \\
\text { 13, no. } 1 \text { (Manila: De La Salle University Press, 2013). }\end{array}$ & 0 \\
\hline & Mga Akda na Hindi Nakalagay sa Google Scholar & \\
\hline & $\begin{array}{l}\text { 1. "Frank Tipler's the Physics of Immortality" (Karunungan, } \\
\text { 1999) }\end{array}$ & \\
\hline & $\begin{array}{l}\text { 2. La Notion de la Liberte Participee dans la Philosophie de Louis } \\
\text { Lavelle (Fribourg: Editions Universitaires Fribourg Suisse, } \\
\text { 1969) }\end{array}$ & \\
\hline & 3. "Pro Death Penalty: Hic et Nunc" (Karunungan, 2000) & \\
\hline & 4. "What is Philosophy?" (Karunungan, 1998) & \\
\hline & 5. Ang Kasaysayan ng Pilosopiya (Zone Print, 1974) & \\
\hline & 6. Ang Pilosopiya ng Tao (Alemar, 1980) & \\
\hline & 7. Ang Pilosopiya ng Diwang Pilipino (United Publishing, 1972) & \\
\hline & 8. Critique of Historical Theory (DLSU, 2002) & \\
\hline & $\begin{array}{l}\text { 9. Festchrift in Honor of Dr. Marcelino Foronda, Jr. (Edited, } \\
\text { DLSU, 1987) }\end{array}$ & \\
\hline & 10. "God in the Third Millenium" (Karunungan, 2002) & \\
\hline & 11. "Hermeneutics of Jurgen Habermas" (Sophia, 1990) & \\
\hline & 12. "Introduction to Hermeneutics" (Karunungan, 2000) & \\
\hline & $\begin{array}{l}\text { 13. “Ang Konsepto ng Kalayaan ni Louis Lavelle" } \\
\text { (Karunungan, 1989) }\end{array}$ & \\
\hline & 14. The Philosophers of Hermeneutics (DLSU, 1990) & \\
\hline & 15. “A New Concept of Philosophy” (Karunungan, 2003) & \\
\hline & 16. "Philosophy as a Critique of Society" (Karunungan, 2002) & \\
\hline & $\begin{array}{l}\text { 17. “Pulong-isip: Meeting of Filipino Minds” (Karunungan, } \\
\text { 1986) }\end{array}$ & \\
\hline & 18." Reflections on the Death of God" (Unitas, 1971) & \\
\hline & $\begin{array}{l}\text { 19. "The Will and Its Relation to Divine Casuality and } \\
\text { Knowledge" (MA Thesis, 1956) }\end{array}$ & \\
\hline \multirow[t]{7}{*}{ 6. Florentino Timbreza } & 1. Pilosopiyang Pilipino (Manila: Rex Book Store, 1982). & 10 \\
\hline & $\begin{array}{l}\text { 2. Alternative to a Dead God (Manila: De La Salle University } \\
\text { Press, 2001). }\end{array}$ & 8 \\
\hline & $\begin{array}{l}\text { 3. Filipino Values Today (Mandaluyong City: National } \\
\text { Bookstore, 2003). }\end{array}$ & 7 \\
\hline & $\begin{array}{l}\text { 4. Filipino Philosophy Today: A Sourcebook in Teaching Filipino } \\
\text { Philosophy (Mandaluyong City: National Bookstore, 2008). }\end{array}$ & 5 \\
\hline & $\begin{array}{l}\text { 5. Bioethics and Moral Decisions (Manila: De La Salle University } \\
\text { Press, 1993). }\end{array}$ & 3 \\
\hline & $\begin{array}{l}\text { 6. Quest for Meaning: Philosophy Made Easy for Filipinos (Manila: } \\
\text { New Day Publishers, 2000). }\end{array}$ & 2 \\
\hline & $\begin{array}{l}\text { 7. Intelektwalisasyon ng Pilosopiyang Filipino (Manila: De La } \\
\text { Salle University Press, 1999). }\end{array}$ & 2 \\
\hline
\end{tabular}




\section{INTERVIEW WITH ROMUALDO ABULAD}

\begin{tabular}{|c|c|c|}
\hline & $\begin{array}{l}\text { 8. “Understanding Filipino Philosophy” in } \\
\text { Karunungan (Manila: University of Santo Tomas Publishing } \\
\text { House, 1997), 9-35. }\end{array}$ & 2 \\
\hline & $\begin{array}{l}\text { 9. Mga Hugis ng Kaisipang Pilipino (Manila: Rex Book Store, } \\
\text { 1989). }\end{array}$ & 2 \\
\hline & $\begin{array}{l}\text { 10. Ensayklopidiya ng Pilosopiya (co-authors: Abulad, Quito, } \\
\text { Reyes), De La Salle University Press, } 1993 .\end{array}$ & 2 \\
\hline & $\begin{array}{l}\text { 11. Logic Made Simple (Quezon City: Phoenix Publishing, } \\
\text { 1992). }\end{array}$ & 1 \\
\hline & $\begin{array}{l}\text { 12. "Sex Selection: Any Taker" in Karunungan, vol. 9, no. } 1 \\
\text { (Manila: University of Santo Tomas Publishing House, 1991). }\end{array}$ & 0 \\
\hline & $\begin{array}{l}\text { 13. Pro Life and Pro Choice Views on Contraception and Abortion } \\
\text { (Manila: De La Salle University Press, 1994). }\end{array}$ & 0 \\
\hline & $\begin{array}{l}\text { 14. “The Filipino Value of Non-violence”, in Filipino Cultural } \\
\text { Traits: Claro R. Ceniza Lectures } 4 \text { (Manila: De La Salle } \\
\text { University Press, 2005). }\end{array}$ & 0 \\
\hline & $\begin{array}{l}\text { 15. "Maituturo ba ang Pagpapahalaga?" in IDEYA: Journal of } \\
\text { the Humanities, vol. 2, no. } 2 \text { (Manila: De La Salle University } \\
\text { Press, 2001). }\end{array}$ & 0 \\
\hline & $\begin{array}{l}\text { 16. “Pamimilosopiya sa Sariling Wika: Mga Problema at } \\
\text { Solusyon", in Malay, vol. 22, no. } 1 \text { (Manila: De La Salle } \\
\text { University Press, 2009), 69-83. }\end{array}$ & 0 \\
\hline & $\begin{array}{l}\text { 17. “Ano ba ang Buhay?", in Malay, vol. 9, no. } 1 \text { (Manila: De } \\
\text { La Salle University Press, 1991). }\end{array}$ & 0 \\
\hline & $\begin{array}{l}\text { 18. Ang Tao Te Ching ni Lao Tzu sa Filipino (Manila: De La } \\
\text { Salle University Press, 1999). }\end{array}$ & 0 \\
\hline & $\begin{array}{l}\text { 19. “Mga Suliranin at Iba Pang Mga Balakid sa Paggamit ng } \\
\text { Filipino sa Pagtuturo", in Malay, vol. 6, no. } 1 \text { (Manila: De La } \\
\text { Salle University Press, 1987). }\end{array}$ & 0 \\
\hline & $\begin{array}{l}\text { 20. “Ulat Tungkol sa Sanggunian at Leksikon ng Pilosopiya” } \\
\text { (co-authors: Quito, Abulad, Reyes), in Malay, De La Salle } \\
\text { University, vol. 3, no. } 2 \text { (July 1983), 157-173. }\end{array}$ & 0 \\
\hline & $\begin{array}{l}\text { 21. “Mga Sangkap ng Pilosopiya ng Buhay ni Huseng Batute", } \\
\text { in Daloy, vol. 4, no. } 2 \text { (Manila: De La Salle University Press, } \\
\text { 1995). }\end{array}$ & 0 \\
\hline & $\begin{array}{l}\text { 22. Paghahanap ng Kabuluhan (Manila: De La Salle University } \\
\text { Press, 2002). }\end{array}$ & 0 \\
\hline & $\begin{array}{l}\text { 23. “Pagsasakatutubo at ang Katutubong Teolohiya”, in } \\
\text { Malay, vol. 5, no. } 1 \text { (Manila: De La Salle University Press, } \\
\text { 1986). }\end{array}$ & 0 \\
\hline & $\begin{array}{l}\text { 24. "Ekolohiya at ang Landasin ng mga Tao", in Malay, vol. } \\
\text { 15, no. } 2 \text { (Manila: De La Salle University Press, 1999). }\end{array}$ & 0 \\
\hline & $\begin{array}{l}\text { 25. "Pilosopiya ng Buhay ng mga Pilipino", in DLSU Graduate } \\
\text { Journal (Manila: De La Salle University Press, 1990). }\end{array}$ & 0 \\
\hline & $\begin{array}{l}\text { 26. “Mga Ugaling Pilipino”, in Malay, vol. 10, no. } 2 \text { (Manila: } \\
\text { De La Salle University Press, 1992). }\end{array}$ & 0 \\
\hline
\end{tabular}




\section{E. DE LEON 35}

\begin{tabular}{|c|c|c|}
\hline & $\begin{array}{l}\text { 27. "Walang Kaibigan, Walang Kumpare, at Walang Kamag- } \\
\text { anak", in IDEYA: Journal of the Humanities, vol. 3, no. } 1 \\
\text { (Manila: De La Salle University Press, 2001). }\end{array}$ & 0 \\
\hline & $\begin{array}{l}\text { 28. "Diwang Pilipino: Pangako at Pagkatao", in Malay, vol. 3, } \\
\text { no. } 2 \text { (Manila: De La Salle University Press, 1984). }\end{array}$ & 0 \\
\hline & 29. “The Marxist Philosophy of Man” (1989) & 0 \\
\hline & $\begin{array}{l}\text { 31. The Florentino T. Timbreza Reader, by Alejandro Padilla } \\
\text { (Manila: De La Salle University Press, 2004). }\end{array}$ & 0 \\
\hline & Mga Akda na Hindi Nakalagay sa Google Scholar & \\
\hline & $\begin{array}{l}\text { 1. "Ang Pagtatanghal sa Bayani at Kabayanihan" } \\
\text { (Karunungan, 1997) }\end{array}$ & \\
\hline & 2. “Ang Sariling Disiplina” (Karunungan, 1999) & \\
\hline & 3. “Ano nga ba ang Pilosopiya?” (Karunungan, 1998) & \\
\hline & $\begin{array}{l}\text { 4. “Can There Be Morality Without Religion?" (Karunungan, } \\
\text { 1998) }\end{array}$ & \\
\hline & 5. “Filipino Concept of Life and Death" (Karunungan, 1986) & \\
\hline & $\begin{array}{l}\text { 6. “Filipino Logic: A Preliminary Analysi” (Karunungan, } \\
\text { 1989) }\end{array}$ & \\
\hline & $\begin{array}{l}\text { 7. "Filipino Philosophy in Dialogue with Three Philosophers } \\
\text { of Non-violence" (Karunungan, 1990) }\end{array}$ & \\
\hline & 8. "Sex, Freedom, and Responsibility" (Sophia, 1986) & \\
\hline & $\begin{array}{l}\text { 9. "The Struggle for Wholeness in Asia: Challenge to } \\
\text { Education in the Next Millenium" (Sophia, 1989) }\end{array}$ & \\
\hline & 10. “Tolerance in Filipino Experience” (Karunungan, 1997) & \\
\hline & 11. “Truth is Greater Friend” (Sophia, 1995-1996) & \\
\hline & 12. Ang Landas ng Buhay ayon kay Lao Tzu (Dissertation, 1980) & \\
\hline & 13. “Filipino Perception of God" (Karunungan, 2002) & \\
\hline & $\begin{array}{l}\text { 14. "The Future of Democracy in the Philippines" (New York: } \\
\text { Journal of Philosophy, 1988) }\end{array}$ & \\
\hline & $\begin{array}{l}\text { 15. “Konseptong Pangkasamaan ni Santo Tomas de Aquino" } \\
\text { (Karunungan, 1989) }\end{array}$ & \\
\hline & 16. “Man and Morality" (Karunungan, 1999) & \\
\hline & 17. “Moral Issue of Abortion” (Sophia, 1991) & \\
\hline & $\begin{array}{l}\text { 18. "The Moral Issue of Lovemaking Without Baby Making" } \\
\text { (Sophia, 1993-1994) }\end{array}$ & \\
\hline & 19. “Parusang Kamatayan” (Karunungan, 2000) & \\
\hline & $\begin{array}{l}\text { 20. “Pilosopiya Bilang Tagasuri ng Kulturang Filipino" } \\
\text { (Karunungan, 2002) }\end{array}$ & \\
\hline & 21. Pilosopiyang Pilipino (Rex Book Store, 1982) & \\
\hline & 22. Sariling Wika at Pilosopiyang Filipino (C\&E, 2008) & \\
\hline & $\begin{array}{l}\text { 23. "Shinto Religion and the Japanese Way of Life" (Sophia, } \\
\text { 1990) }\end{array}$ & \\
\hline \multirow[t]{4}{*}{ 7. Magdalena Villaba } & 1. The Mission of Women: A Return to their Original Role (1975) & 0 \\
\hline & 2. “Confucian Humanism” (Unitas, 1993) & 0 \\
\hline & Mga Akda na Hindi Nakalagay sa Google Scholar & \\
\hline & 1. "Phenomena and Their Interpretation" (Unitas, 1993) & \\
\hline
\end{tabular}

(c) 2016 Emmanuel C. De Leon

http://www.kritike.org/journal/issue 18/de leon june2016.pdf

ISSN 1908-7330 


\section{INTERVIEW WITH ROMUALDO ABULAD}

\begin{tabular}{|c|c|c|}
\hline & $\begin{array}{l}\text { 2. “Ang Wikang Filipino sa Kasaganaan at Kapayapaan” } \\
\text { (Unitas, 1999) }\end{array}$ & \\
\hline & $\begin{array}{l}\text { 3. "The Concept of Maya in the Philosophy of Shankara" } \\
\text { (Karunungan, 2000) }\end{array}$ & \\
\hline & 4. “The Dominican's Mission Here and Now” (Unitas, 1988) & \\
\hline & 5. Philosophy of the East (UST Publishing House, 1996) & \\
\hline & 6. Prelude to the Wisdom of the East & \\
\hline & $\begin{array}{l}\text { 7. "The Social Philosophy of Mahatma Gandhi" (UST Journal } \\
\text { of Graduate Research, 1988) }\end{array}$ & \\
\hline \multirow[t]{18}{*}{ 8. Manuel Pinon } & NONE & NONE \\
\hline & Mga Akda na Hindi Nakalagay sa Google Scholar & \\
\hline & $\begin{array}{l}\text { 1. "An Evaluation of the Reply to the Questionnaire of the } \\
\text { Population Institute" (Santo Tomas Journal of Medicine, 1970) }\end{array}$ & \\
\hline & $\begin{array}{l}\text { 2. Being and Reality: The Philosophy of the Contingent Being and } \\
\text { Contingent Reality (UST Press, 1972) }\end{array}$ & \\
\hline & $\begin{array}{l}\text { 3. Birth Control and Family Planning in the Light of 'Humanae } \\
\text { Vitae'" }\end{array}$ & \\
\hline & 4. "Change and Reform” (Unitas, 1972) & \\
\hline & 5. Elements of Metaphysics (UST Press, no date) & \\
\hline & $\begin{array}{l}\text { 6. "Fertilization of in Vitro, Its Morality" (Santo Tomas } \\
\text { Journal of Medicine, 1970) }\end{array}$ & \\
\hline & $\begin{array}{l}\text { 7. Fundamentalism: Bible Christians Rejection of the Blessed Virgin } \\
\text { Mary and Born-again Christian (RTP Foundation, 1989) }\end{array}$ & \\
\hline & $\begin{array}{l}\text { 8. Judgment on Agrarian Reformatory Expropriations: A } \\
\text { Sociological, Economic, Historical, Juridical, and Ethical Study } \\
\text { (UST, 1959) }\end{array}$ & \\
\hline & $\begin{array}{l}\text { 8. A Magna Carta for the Unborn: Fifth Commandment Thou Shall } \\
\text { Not Kill (RTP Foundation, 19?) }\end{array}$ & \\
\hline & $\begin{array}{l}\text { 9. The Metaphysical Conciliation of the Universal Primary } \\
\text { Causality of God with the Particular Secondary Causality of } \\
\text { Creatures in Particular with the Free Action of the Will (UST } \\
\text { Press, 1971) }\end{array}$ & \\
\hline & $\begin{array}{l}\text { 10. "'Musings a Propus' the Population Year and Population } \\
\text { Education" (Boletin Eclesiatico de Filipinas, 1974) }\end{array}$ & \\
\hline & $\begin{array}{l}\text { 11. “The Natural Right of Personality (Does the Unborn } \\
\text { Human Fetus have such a Right?)" (Boletin Eclesiastico de } \\
\text { Filipinas, 1978) }\end{array}$ & \\
\hline & $\begin{array}{l}\text { 12. The Nature and Causes of the Psychological Freedom or } \\
\text { Psychical Mastery of the Will Over Its Acts in the Writings of St. } \\
\text { Thomas (undated) }\end{array}$ & \\
\hline & $\begin{array}{l}\text { 13. Off-beat Aggiornamento: The New Prophetic Movement and the } \\
\text { New Charismatic Church (Manila: Novel, 1969) }\end{array}$ & \\
\hline & $\begin{array}{l}\text { 14. Predestination and Salvation (QC: Dominacan House of } \\
\text { Studies, 1977) }\end{array}$ & \\
\hline & $\begin{array}{l}\text { 15. Psychological Freedom: Mastery of the Will Over Its Acts Form } \\
\text { and Under Efficacious Grace (RTP Foundation, 1987) }\end{array}$ & \\
\hline
\end{tabular}




\section{E. DE LEON 37}

\begin{tabular}{|l|l|l|}
\hline & 16. Religion and "Religions" (Dominian House of Studies, 1980) & \\
\hline & $\begin{array}{l}\text { 17. Review Article "Musing on 'Inculturation' and the } \\
\text { Filipinization of Theology" (Unitas, 1981) }\end{array}$ & $\begin{array}{l}\text { 18. "A Study on 'A Study of Sin'” (Boletin Eclesiastico de } \\
\text { Filipinas, 1978) }\end{array}$ \\
\hline & $\begin{array}{l}\text { 19. “Teaching of the Church on the Carp and the Natural } \\
\text { Right to Ownership" (Boletin Eclesiastico de Filipinas, 1989) }\end{array}$ & \\
\hline
\end{tabular}

Department of Philosophy, University of Santo Tomas, Philippines 